OPEN ACCESS

Edited by:

Safikur Rahman,

Munshi Singh College, Babasaheb

Bhimrao Ambedkar Bihar University,

India

Reviewed by:

Md Tabish Rehman,

King Saud University, Saudi Arabia

Nitesh Kumar Poddar,

Invertis University, India

*Correspondence:

Avinash Kale

avinash.kale@cbs.ac.in

Received: 04 August 2020 Accepted: 09 September 2020

Published: 06 October 2020

Citation:

Pathak S, Parkar H, Tripathi S and Kale A (2020) Ofloxacin as a Disruptor of Actin Aggresome "Hirano Bodies": A Potential Repurposed Drug for the

Treatment of Neurodegenerative

Diseases.

Front. Aging Neurosci. 12:591579.

doi: 10.3389/fnagi.2020.591579

\section{Ofloxacin as a Disruptor of Actin Aggresome "Hirano Bodies": A Potential Repurposed Drug for the Treatment of Neurodegenerative Diseases}

\author{
Samridhi Pathak, Haifa Parkar, Sarita Tripathi and Avinash Kale* \\ School of Chemical Sciences, University of Mumbai - Department of Atomic Energy Center for Excellence in Basic Sciences, \\ University of Mumbai, Vidyanagari Campus, Mumbai, India
}

There is a growing number of aging populations that are more prone to the prevalence of neuropathological disorders. Two major diseases that show a late onset of the symptoms include Alzheimer's disorder (AD) and Parkinson's disorder (PD), which are causing an unexpected social and economic impact on the families. A large number of researches in the last decade have focused upon the role of amyloid precursor protein, $A \beta$-plaque, and intraneuronal neurofibrillary tangles (tau-proteins). However, there is very few understanding of actin-associated paracrystalline structures formed in the hippocampus region of the brain and are called Hirano bodies. These actin-rich inclusion bodies are known to modulate the synaptic plasticity and employ conspicuous effects on long-term potentiation and paired-pulse paradigms. Since the currently known drugs have very little effect in controlling the progression of these diseases, there is a need to develop therapeutic agents, which can have improved efficacy and bioavailability, and can transport across the blood-brain barrier. Moreover, finding novel targets involving compound screening is both laborious and is an expensive process in itself followed by equally tedious Food and Drug Administration (FDA) approval exercise. Finding alternative functions to the already existing FDA-approved molecules for reversing the progression of age-related proteinopathies is of utmost importance. In the current study, we decipher the role of a broad-spectrum general antibiotic (Ofloxacin) on actin polymerization dynamics using various biophysical techniques like right-angle light scattering, dynamic light scattering, circular dichroism spectrometry, isothermal titration calorimetry, scanning electron microscopy, etc. We have also performed in silico docking studies to deduce a plausible mechanism of the drug binding to the actin. We report that actin gets disrupted upon binding to Ofloxacin in a concentration-dependent manner. We have inferred that Ofloxacin, when attached to a drug delivery system, can act as a good candidate for the treatment of neuropathological diseases.

Keywords: ofloxacin, neurodegenerative diseases, actin, repurposable drugs, biophysical studies, SEM, in silico 


\section{INTRODUCTION}

Neurodegenerative disorders (NDs) are the subset of brain disorders defined by the obliteration of neuronal cells resulting from the accumulation of protein aggregates (Perl et al., 1995). Deaths related to NDs are second-most around the globe and a prominent cause of disability worldwide (Feigin et al., 2019). An estimated 12 million Americans will be suffering from NDs by 2030, of which patients suffering from Alzheimer's disease would be on top of the list (Chanfreau et al., 2005; Gammon, 2014). The biological factors that are known to cause NDs are attributed to oxidative stress, cytoskeletal protein aggregation, abnormal ubiquitination of protein, mitochondrial dysfunction, etc. (Trends and Disorders, 2018). Diseases like Alzheimer's disorder (AD), Parkinson's disease (PD), Huntington's disease (HD), amyotrophic lateral sclerosis (ALS), frontal temporal dementia (FTD) falls under the category of NDs and a major contributor to the socioeconomic problems associated with it (Subramaniam, 2019). Various factors have been associated to be the causative agent behind NDs, but a prominent one is still far from the search. Depending on the type of NDs, it could be either familial or sporadic (Przedborski et al., 2003). The current treatment regime focuses on slowing the manifestations of the symptoms and providing temporary relief toward these symptoms, thus, causing a severe lack of procedure to slow the disease progression and eventual death (Trends and Disorders, 2018).

Cytoskeletal protein has a major role to play in neuronal functioning by providing flexibility and maintaining the neuronal circuit (Muñoz-Lasso et al., 2020b). Certain proteins that are widely studied for their role in NDs are prion, tau, $\beta$-amyloid, $\alpha$-synuclein, and Huntington (Gitler et al., 2017). It is implicated that misfolding of these proteins leads to their aggregation in the neuronal cells resulting in neurodegeneration (Ross and Poirier, 2004; Santa-Mara et al., 2008; Goebel, 2009; Spears et al., 2014; Koistinen et al., 2017). However, the role of cytoskeletal protein actin in the manifestation of NDs have been far from understood. Actin is reported to be the driving force in controlling synaptic plasticity and maintaining the structural integrity of the synapses (Kim and Lisman, 1999; Luo, 2002; Fukazawa et al., 2003; Bourne and Harris, 2008; Okada and Soderling, 2009). It functions by changing its morphology in response to different types of neural activity (Svennberg, 2006; Gordon-Weeks and Fournier, 2014; Spence and Soderling, 2015; Szabó et al., 2016; Pelucchi et al., 2020). Defects in the regulation of actin protein is one of the contributing factors leading to neurological disorders (Kim and Lisman, 1999; Luo, 2002; Bourne and Harris, 2008; Okada and Soderling, 2009).

Actin cytoskeletal remodeling and rearrangement play an important role at different sites in the brain cells and different stages of brain activity (Kevenaar and Hoogenraad, 2015; Yamada and Kuba, 2016). In the axonal cells, actin occurs as a meshwork of branched filaments (Yamada and Kuba, 2016). During polarization of neurons, actin and its regulators control the assembly and disassembly of F-actin filament in order to regulate the axonal elongation and contribute to the formation of axonal filopodia (Kevenaar and Hoogenraad, 2015). Aberrations in the axonal cytoskeletal-dependent process lead to defects in axonal transport, outgrowth, targeting, and synapse functioning, which, in turn, is associated with ALS (Kevenaar and Hoogenraad, 2015). In the mature neurons, actin is associated with regulating the presynaptic functions such as conscripting and repositioning of a synaptic vesicle, maintaining exocytosis and endocytosis. Mis-regulated actin in mature neurons has been implicated in various mental illnesses such as intellectual disability and schizophrenia (Ogawa and Rasband, 2008). Actin polymerization dynamics has a major role in sustaining the morphology of the spine and is associated with long-term memory via activation of long-term potentiation (LTP) or long-term depression (LTD) of excitatory signal transmission (Kim and Lisman, 1999; Luo, 2002; Bourne and Harris, 2008; Okada and Soderling, 2009). Also, actin rods are involved in the progression of axonal sensory neuropathy: a condition where neurons are damaged as observed in frataxin-deficient dorsal root ganglion (DRG) neurons (Muñoz-Lasso et al., 2020a).

The presence of F-actin aggregates has also been found in Hirano bodies, which are one of the causes of AD (Sabo et al., 2001; Maselli et al., 2003; Melidone et al., 2011; Furgerson et al., 2012; Yao and Khan, 2013). Hirano bodies are cytoplasmic inclusion bodies, rod-shaped eosinophilic in nature. It was first found in the hippocampus region of the aged brain in patients suffering from ALS and PD (Goldman, 1983; Sabo et al., 2001; Maselli et al., 2003; Melidone et al., 2011; Furgerson et al., 2012; Yao and Khan, 2013). The protein aggregate was filamentous, paracrystalline in nature, and thin filaments of $6 \mathrm{~mm}$ in size were observed. Actin depolymerization factor (ADF)/cofilin, an actinbinding protein, which helps sever F-actin, is severely reduced under stress conditions thereby enhancing the formation of aggregates (Yang et al., 2010; Muñoz-Lasso et al., 2020a,b). This leads to the development of rod-like structures of actin filaments widely present in neurons of patients with $\mathrm{AD}$ and $\mathrm{PD}$ (Furukawa and Fechheimer, 1997; Yang et al., 2010).

Owing to the aforementioned problems associated with actin misfolding, dysregulation, and its subsequent aggregation, it can form a potential therapeutic target for neurological disorders. A previous study by Pathak et al. (2020) elucidates the effect of the tetracycline group of antibiotics on F-actin protein aggregate. It has paved a way to think toward the detection of targeted therapies against F-actin aggregates. Due to this, the use of Food and Drug Administration (FDA)-approved drugs could form a potential alternative to act against actin aggregates responsible for neurodegenerative and neurodevelopmental disorders. Drug repositioning has become one of the widely used approaches in recent times, as it is highly efficient, economical, and less vulnerable. This may have a major impact on the reduction of financial burden, the progression of the disease, and subsequently lower the time taken for the drug discovery (Eira et al., 2016; Pushpakom et al., 2019; Pathak et al., 2020).

Ofloxacin is a broad-spectrum antibiotic widely used to treat infections caused due to Staphylococcus aureus, methicillinresistant Staphylococcus aureus (MRSA), Streptococcus spp., Enterococcus faecalis, Enterobacteriaceae, and Pseudomonas aeruginosa. It comes under the class of fluoroquinolones and structurally, it is a tricyclic ring that has a methyl group 
being attached to the C-3 position (asymmetric carbon) on the oxazine ring (Morrissey et al., 1996). Owing to the safety of this drug, which has passed all the clinical trial phases, our study has identified it as a molecular entity with a new function. In the current study, we have investigated the role of Ofloxacin on F-actin aggregates using various biophysical techniques such as right-angle light scattering (RLS), dynamic light scattering (DLS), circular dichroism spectroscopy (CD), and kinetics study. The imaging studies using scanning electron microscopy (SEM) were also carried out, and the plausible mode of binding of Ofloxacin to F-actin protein was studied using isothermal titration calorimetry (ITC) and in silico studies. We have proposed a plausible mode of binding of Ofloxacin to actin and its subsequent disruption into the smaller oligomeric state. We thus propose that actin aggregates can be broken into smaller oligomers and be made available for new rounds of polymerization using Ofloxacin, if we have a proper drug delivery system in place, which is capable of crossing the bloodbrain barrier (BBB).

\section{MATERIALS AND METHODS}

\section{Purification and Characterization of Actin}

All chemicals used for the experiment were procured from S.D Fine (Mumbai, India) except for DTT and Ofloxacin (PubChem CID: 4583), which were procured from Sigma Aldrich (Mumbai, India). Acetone powder from pig thigh muscle (Sus scrofa domesticus) was prepared following a standard protocol (Solomon et al., 2010). Actin was purified using the method developed by Spudich et al. in the year 1971 for rabbit skeletal muscle (Solomon et al., 2010) with minor modifications. In brief, acetone powder was homogenized in G-actin buffer (GB) (composition: $2 \mathrm{mM}$ Tris- $\mathrm{HCl}, 0.2 \mathrm{mM}$ ATP, $0.5 \mathrm{mM}$ DTT, and $0.2 \mathrm{mM} \mathrm{CaCl}_{2}$ ) followed by treatment with polymerization buffer (PB: Composition: $800 \mathrm{mM} \mathrm{KCl}$ and $5 \mathrm{mM} \mathrm{MgCl}_{2}$ ). It was then kept for overnight polymerization with constant stirring at $4^{\circ} \mathrm{C}$, followed by ultracentrifugation at 75,000 rpm for $90 \mathrm{~min}$. The ultracentrifuge used was a tabletop model from Beckman Coulter XP-100 at Bombay College of Pharmacy (BCP), Mumbai, India. Purified polymer actin (F-actin) was characterized on MALDITOF (Bruker Daltonik GmbH) (Chavan et al., 2016) at the Advanced Centre for Treatment, Research, and Education in Cancer (ACTREC), Navi Mumbai, India. In order to exchange the solvent system from PB to either GB or water, the F-actin pellet was resuspended in the respective solvent and was dialyzed against the desired solvent system for $72 \mathrm{~h}$ at $4^{\circ} \mathrm{C}$ with buffer change every $12 \mathrm{~h}$.

\section{Light-Scattering Measurements}

To avoid any interference in the scattering analysis, independent constant wavelength synchronous fluorescence (CWSF) measurements were carried out on actin and Ofloxacin in the polymerization buffer system. All the aforementioned compounds were procured from Himedia (Mumbai, India) and Sigma Aldrich. All these measurements were carried out on Cary Eclipse Fluorescence Spectrophotometer from Agilent technologies with the following parameters while keeping the difference between excitation and emission wavelength $(\Delta \lambda)$ at zero: excitation wavelength: $200-700 \mathrm{~nm}$, emission wavelength: 200-700 nm, excitation slit width: 5, emission slit width: 5, excitation filter: auto, emission filter: auto, temperature: room temperature, time points: T0, T3, T6, T24, T48, and T72 h. It should be noted that we have shown data only up to $48 \mathrm{~h}$ for clarity purposes. The concentration of actin was fixed at $3 \mu \mathrm{M}$, whereas, for Ofloxacin, measurements were carried out for the highest concentration of 3,000 $\mu \mathrm{M}$ to ensure that there is no concentration-dependent aggregation of the compound. The synchronous curves are depicted in Figure 1. Likewise, measurements were also carried out for water and G-actin buffer systems.

Based on the findings of the synchronous measurements, all the scattering experiments were carried out using the following parameters: excitation wavelength: $350 \mathrm{~nm}$, emission wavelength: $350 \mathrm{~nm}$, excitation slit width: 5, emission slit width: 5, excitation filter: auto, emission filter: auto. The concentration of actin protein was kept constant at $3 \mu \mathrm{M}$ and was incubated with varying concentrations $(3,30,150,300,750,1,500$, and 3,000 $\mu \mathrm{M})$ of Ofloxacin. Scattering measurements were carried out at the following time points: T0, T3, T6, T12, T18, T24, T36, T48, and $\mathrm{T} 72 \mathrm{~h}$. These measurements were done in the three different solvent systems, namely, PB, GB, and water.

\section{Dynamic Light Scattering Studies}

The effect of Ofloxacin on the oligomeric state and heterogeneity of F-actin was measured using DLS. The experiments were performed on Malvern Panalytical (United Kingdom) at the Department of Biophysics, University of Mumbai, India. The position of the attenuator was set at 4.65 , which was determined automatically based on the size and the concentration of the actin polymer in association with the respective compounds. The samples were taken in a polystyrene disposable sizing cuvette, and folded capillary zeta cell was used for the measurement of zeta potential. F-actin was prepared in PB buffer, and the concentration was kept constant at $3 \mu \mathrm{M}$. The concentration of Ofloxacin was maintained at $300 \mu \mathrm{M}$ (compound ratio to actin was kept at 1:100). At least two measurements were carried out for each of the interactions between F-actin and the drug. A detection angle of $90^{\circ}$ was used for the measurement of the size. The analysis was carried out on a zeta sizer Nano-ZS90 DLS system at $25^{\circ} \mathrm{C}$, equipped with red $(633 \mathrm{~nm})$ laser and avalanche photodiode detector (quantum efficiency $>50 \%$ at $633 \mathrm{~nm}$ ). The software associated with this DLS machine was Dispersion Technology Software (DTS) version 7.0, which was used to analyze the $\mathrm{z}$-average hydrodynamic radius, intensity distribution, volume distribution, polydispersity index (PDI), autocorrelation function, and zeta potential (Liu et al., 2012).

\section{Circular Dichroism Spectroscopy}

The changes in the secondary structure of F-actin in the three different solvent systems, namely, PB, GB, and water were monitored using CD spectroscopy. All measurements were carried out between 260 and $200 \mathrm{~nm}$ using a JASCO-J-815 

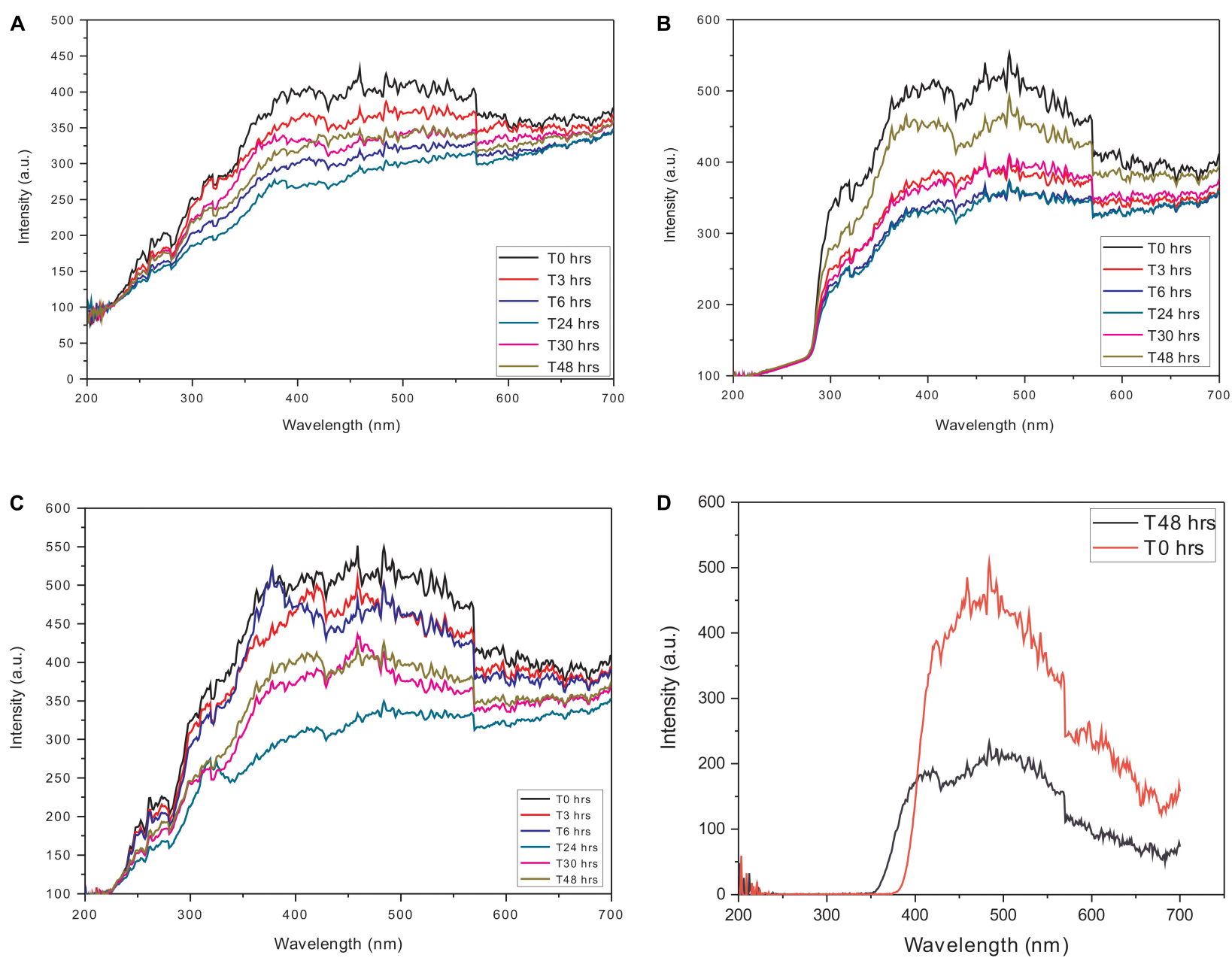

FIGURE 1 | Constant wavelength synchronous analysis (CWSF) profile for actin control in (A) polymerization buffer (PB), (B) G-actin buffer (GB), (C) water, and (D) Ofloxacin control in PB. Black color represents CWSF at $0 \mathrm{~h}$, red color represents CWSF at $3 \mathrm{~h}$, blue color represents $\mathrm{CWSF}$ at $6 \mathrm{~h}$, green color represents CWSF at $24 \mathrm{~h}$, pink color represents CWSF at $30 \mathrm{~h}$, and the cyan color represents CWSF at T48 $\mathrm{h}$.

spectropolarimeter at the National Centre for Cell Sciences (NCCS), Pune, India. The protein was prepared in the respective buffer, and the concentration was fixed at $5 \mu \mathrm{M}$. The ratio of protein:drug Ofloxacin was taken as 1:5 $(25 \mu \mathrm{M}), 1: 10$ $(50 \mu \mathrm{m}), 1: 20(100 \mu \mathrm{M}) 1: 50(250 \mu \mathrm{M})$, and 1:100 $(500 \mu \mathrm{M})$. The parameters used in the experiment were as follows: the bandwidth was maintained at $1.00 \mathrm{~nm}$, and the cell of $1 \mathrm{~mm}$ path length was used. The data were presented as mean residue ellipticity $[\theta]$ in deg $\mathrm{cm}^{2} \mathrm{dmol}^{-1}$, which is defined by equation 1 below:

$$
[\theta]=C D / 10 \times n \times 1 \times C p)
$$

where $\mathrm{CD}$ is in millidegree, $\mathrm{n}$ is the number of amino acid residues, 1 is the path length of the cell in $\mathrm{cm}$, and $\mathrm{Cp}$ is the molar concentration of the protein (Sonavane et al., 2017). The K2D2 (Perez-Iratxeta and Andrade-Navarro, 2008) software was used, and further analysis of the data was done using CAPITO (Wiedemann et al., 2013).

\section{Imagining Studies Using Scanning Electron Microscopy}

The SEM analysis was carried out on a ZEISS microscope using the following parameters: EHT ( $3 \mathrm{kV})$ and signal (SE2). It should be noted that all the imaging assays were performed in GB buffer, and water as PB buffer was high in salt concentration, thereby, limiting us for imaging in PB buffer. SEM imaging was carried out at the Tata Institute of Fundamental Research (TIFR), Mumbai.

\section{Kinetic Measurements for Actin Aggregation}

The rate of depolymerization of F-actin in the presence and absence of Ofloxacin was performed using different concentrations of drugs ranging from 3 to $90 \mu \mathrm{M}$. The concentration of actin was fixed at $3 \mu \mathrm{M}$ and was prepared in $\mathrm{PB}$. The effect of the drug on the depolymerization kinetics of actin was measured using RLS on spectrofluorimeter (Agilent technology) (Borana et al., 2014). These measurements were 
performed at room temperature with excitation and emission wavelengths fixed at $350 \mathrm{~nm}$. In each case, the slit width for both excitation and emission was kept at $5 \mathrm{~nm}$. The measurements were carried out for $30 \mathrm{~min}$ to ensure the attainment of saturation points for the respective compounds. The above parameter for the kinetic analysis was derived based upon our initial synchronous experiments with the offset value of zero (Borana et al., 2014).

All the kinetic traces were adequately fit to a single exponential decay curve as defined by the following equation (Equation 2):

$$
y=y 0+A 2 \times \exp \left(-\frac{x}{t 1}\right)
$$

where A2 stands for the amplitude, y0 stands for the maximum degradation/depolymerization, and $\mathrm{t} 1$ stands for the time constant. Depolymerization rate constant $\mathrm{K}_{a p p}$ was apparently calculated as the inverse of the apparent rate constant.

$$
K_{a p p}=1 / t
$$

Since all of the traces showed nearly to be a two-phased reaction, the amplitude of the first phase reaction was calculated by the following equation (Equation 3):

$$
A 1=y_{\max }-A 2
$$

where $y_{\text {max }}$ is the RLS value in the absence of drugs.

\section{Isothermal Titration Calorimetric Measurements}

Isothermal titration calorimetric (ITC) experiments were carried out to understand the association constant (Ka), thermodynamic parameters of binding $(\Delta \mathrm{H})$, entropy $(\Delta \mathrm{S})$, and the stoichiometry (n) of the interaction of the drug with F-actin. The protein was prepared in $\mathrm{PB}$ buffer and kept for dialysis for $72 \mathrm{~h}$ at $4^{\circ} \mathrm{C}$ on a magnetic stirrer. All the measurements were carried out on a Malvern MicroCal ITC 200. The following parameters were used for the analysis: the total number of injections for Ofloxacin was 19 , cell temperature was kept at $25^{\circ} \mathrm{C}$, reference power was kept at 10 , the initial delay was 180 , cell concentration (F-actin): $0.067 \mathrm{mM}$, syringe concentration for Ofloxacin was $1.34 \mathrm{mM}(20 \times)$.

\section{In silico Data Analysis}

Actin interaction with that of Ofloxacin was carried out in order to predict the site of binding of the drug to the actin oligomer (Hexamer). For all our analyses, we had used the ADP bound actin monomer (PDB ID: 1J6Z) (Otterbein et al., 2001). The structure was carefully observed and was duly corrected for incomplete residual side chains using the simple mutate function of the WinCoot tool (Crystallographic Object-Oriented Toolkit) (Emsley et al., 2010; Debreczeni and Emsley, 2012). This modified monomer was used to prepare a hexametric polymer using the cryo-EM structure, 3J0S as a template molecule using the SSM superpose function of the WinCoot. Subsequently, the hexamer was energy minimized using the online Gromacs Minimizer 5.0 tool (Abraham et al., 2015). The 3D structure of Ofloxacin was downloaded from PubChem (CID: 4583) and saved as an SDF file. This SDF file was then converted to PDB using the Discovery Studio 2019 Client (BIOVIA Discovery Studio Visualizer).

AutoDockTools-1.5.6 was used to prepare the ".pdbqt" files for both actin polymer and Ofloxacin. AutoDock Vina 4.2.6. was used for carrying out the docking studies of the actin with the drug utilizing the standard search parameters (Trott and Olson, 2010). Obtained results were analyzed using Pymol as a visualization tool.

\section{RESULTS}

\section{Purification and Characterization of Actin}

Our sequence analysis showed that human actin was having more than $90 \%$ similarity to that of pig actin. Actin being highly conserved across the higher eukaryotes, the pig (S. scrofa domesticus) thigh muscle was used for the purification of actin. In order to purify actin, acetone powder was prepared from pig thigh muscle and stored at $-80^{\circ} \mathrm{C}$. Actin was purified using the protocol as mentioned and modified by Pathak et al. (2020) in batches for all our assays. The purified actin had a mass of $42 \mathrm{kDa}$ as observed in our SDS page analysis, which was also confirmed by excising the aforementioned band from the gel and subjecting it to mass spectrometric analysis.

\section{Constant Wavelength Synchronous Analysis}

In order to perform RLS analysis for the actin-Ofloxacin interaction, it was important for us to know the parameters for the same. We performed CWSF for both the drug molecule (Ofloxacin) as well as actin in three different buffer systems viz: $\mathrm{PB}, \mathrm{GB}$, and Water. It was observed in our results (Figures 1A-C) that actin in all the three buffer systems shows a very high scattering from 250 to $700 \mathrm{~nm}$ up to $48 \mathrm{~h}$. Ofloxacin has negligible scattering observed up to $390 \mathrm{~nm}$ beyond, which starts showing a very high scattering as observed in Figure 1D. Owing to our CWSF data, we performed all our right-angle scattering analysis at a wavelength of around $350 \mathrm{~nm}$, as actin in all the three buffer systems showed significant scattering, while Ofloxacin did not show any scattering. This would, hence, avoid any interference from the drug while collecting the data for right-angle scattering.

\section{Right-Angle Light Scattering Measurements}

Actin-Ofloxacin interaction was studied using RLS at the different concentrations for up to $48 \mathrm{~h}$ in the three aforementioned buffer systems. We observed that actin control in buffer systems showed a very high scattering of around $700 \mathrm{~nm}$ in $\mathrm{PB}$ and water and around $450 \mathrm{~nm}$ in GB. This high intensity was directly proportional to the size of the aggregate present in our control systems. Both $\mathrm{PB}$ and water has polymerized filamentous actin as well as aggregated actin, while GB has oligomeric actin present in them indicating the three different morphological states of the protein as found in the in vivo system. Also, there was very little drop in the measured intensity 
of the actin controls with respect to time, which was indicative of actin's intrinsic property to polymerize and depolymerize. Upon treatment with different concentrations of Ofloxacin as observed in Figures 2A-C, there was a proportional drop in the scattering intensity as the function of its concentration as well as the time of treatment. At higher concentrations from 30 to $3,000 \mu \mathrm{M}$, the scattering intensity is very low in all the three buffer systems indicating that Ofloxacin upon interaction with either the polymerized actin, higher oligomeric actin, or aggregated actin gets disintegrated to smaller oligomeric and subsequently to monomeric actin. This interaction is also shown to be irreversible as there was no rise in the scattering intensity even after 48-96 h.

\section{Dynamic Light Scattering for Actin Compound Interaction Studies}

As observed from Figures $\mathbf{3 A}, \mathbf{C}, \mathbf{E}$, actin control in the three solvent systems viz, $\mathrm{PB}, \mathrm{GB}$, and water shows the presence of a heterogeneous population of actin aggregates, which varies in size. The two peaks vary in the size of aggregated actin present in each of the three solvent systems. In $\mathrm{PB}$, the size was deciphered to be around 90-150 d nm for the first peak, from 350 to $800 \mathrm{~d} \mathrm{~nm}$. In GB, the size of the two peaks has been deduced to be $80-120$ and 300-400 $\mathrm{d}$ nm while in water, the size of the two peaks was more than $1,000 \mathrm{~d} \mathrm{~nm}$. This size variance and heterogeneity of the peaks show that actin control has a different morphology in the three different solvent systems. However, upon treatment with Ofloxacin, actin in PB, $\mathrm{GB}$, and water were disintegrated into a homogenous population of smaller monomeric/oligomeric state actin as observed in Figures 3B,D,F respectively. The size of the homogenous peaks was deduced to be 70-80 $\mathrm{d} \mathrm{nm}$ in PB, 450-500 $\mathrm{d} \mathrm{nm}$ in GB, and $100-120 \mathrm{~d} \mathrm{~nm}$ in water.

\section{Spectrophotometric Analysis of the Actin Compound Interaction Studies}

We also performed circular dichroism spectroscopic analysis (CD) for both untreated actin control and treated actin with Ofloxacin in $\mathrm{PB}, \mathrm{GB}$, and water, respectively. The $\mathrm{CD}$ data obtained in mdeg was analyzed using the CAPITO software. As observed in Figures 4A-C, actin control in PB, GB, and water shows the curve to have a single peak dip at $212 \mathrm{~nm}$ indicative of the presence of more polymeric, filamentous, or aggregated structures. However, upon treatment with Ofloxacin, actin in all the three respective buffer system shows the change in the curve profile to contain more $\alpha$-helical structure closer to globular actin monomer with two dips observed at 211 and $220 \mathrm{~nm}$.

Our CAPITO analysis as observed in Figures $4 \mathrm{~A}-\mathrm{C}$ also suggests the compactness of the actin protein upon treatment with different concentrations of Ofloxacin either as globule, molten globule, premolten globule, or unfolded protein. Untreated actin in PB exists as molten globule, which is indicative of compact partially folded conformation with near-native compression, whereas in GB and water, it exists as a globular structure. As soon as we treat actin in the PB buffer system with Ofloxacin at 50 and $100 \mu \mathrm{M}$, a concentration-dependent change in the structural content of the actin was observed. At $50 \mu \mathrm{M}$, the actin oligomer lies between the conformation of the molten globule and globular protein indicating the presence of a larger oligomeric actin with increasingly exposed hydrophobic residues (Perera et al., 2016). However, as soon as the concentration is increased to $100 \mu \mathrm{M}$, actin, which is further disrupted to either small oligomers or monomers, has a compact near-native structure with substantial $\alpha$-helical content and more organized tertiary structure. Similarly, when actin in GB was treated with Ofloxacin, there was a structural change observed with respect to the concentration of the drug molecule. At a lower concentration of $50 \mu \mathrm{M}$, actin is disintegrated into smaller oligomers and monomers prevailing as a molten globular protein with partially exposed hydrophobic residues rich in $\alpha$-helix. However, as soon as the concentration is increased, the partially folded actin protein is switched back to the more globular structure. In water, where the actin exists as an amorphous aggregate, the structural premise of the protein is more globular. As soon as it is treated with Ofloxacin at a concentration of $50 \mu \mathrm{M}$, it occurs as a molten globule and changes to a more globular structure at a higher concentration of $100 \mu \mathrm{M}$. This structural change in actin in water is quite contrary to that observed in actin treated in $\mathrm{PB}$ and $\mathrm{GB}$.

We also analyzed the same set of data using BESTSEL software to calculate the percentage of $\alpha$-helical and $\beta$-structure present in
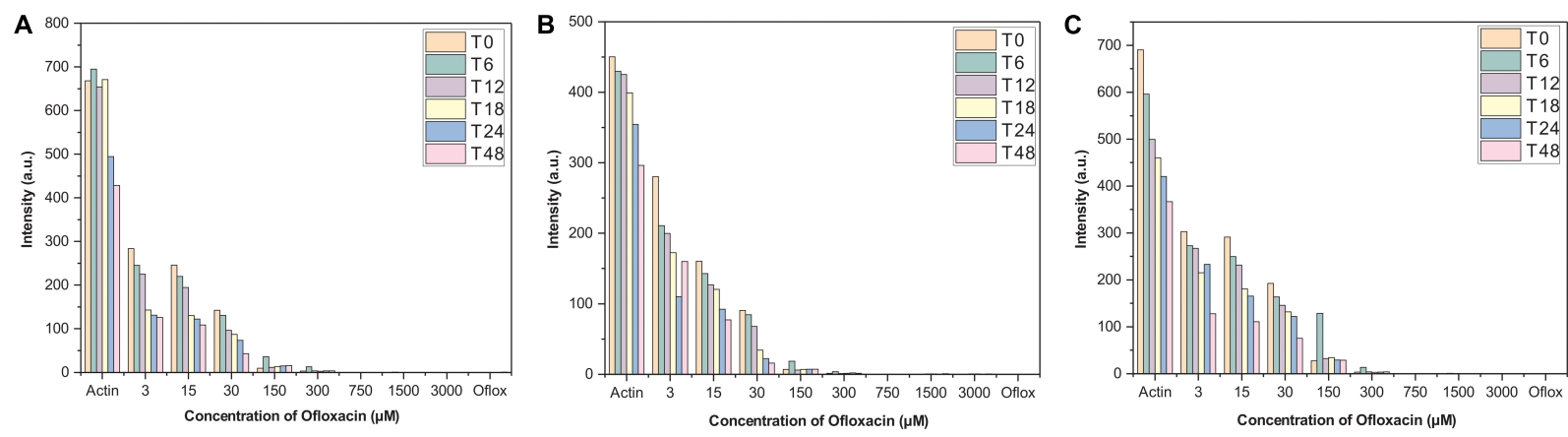

FIGURE 2 | Right-angle light scattering (RLS) profile for actin control and actin treated with Ofloxacin in (A) PB, (B) GB, and (C) water. The orange bar stands for actin aggregates at $0 \mathrm{~h}$, green for $6 \mathrm{~h}$, purple for $12 \mathrm{~h}$, yellow for $18 \mathrm{~h}$, blue for $24 \mathrm{~h}$, and pink for $48 \mathrm{~h}$. 


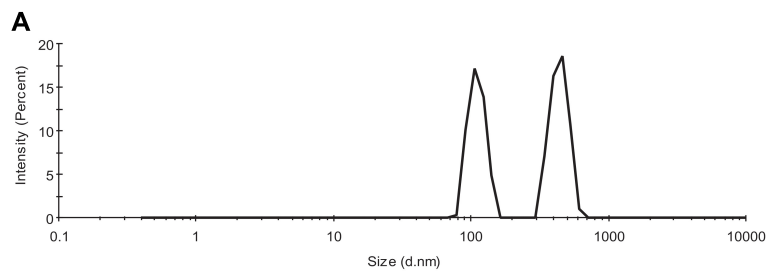

C

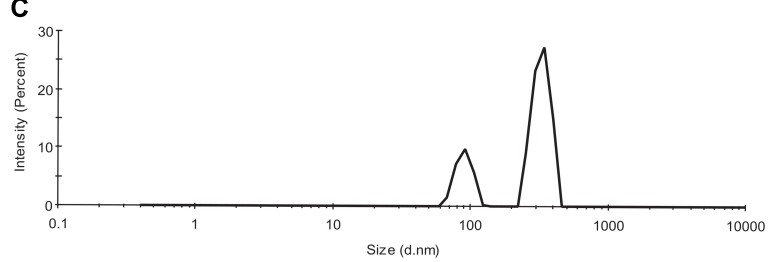

E

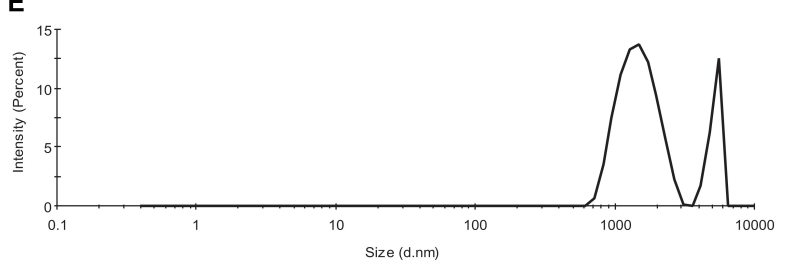

B

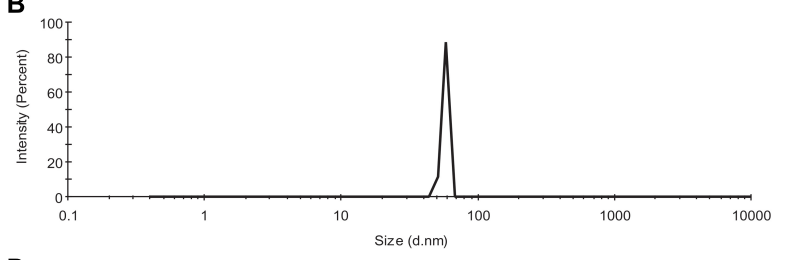

D

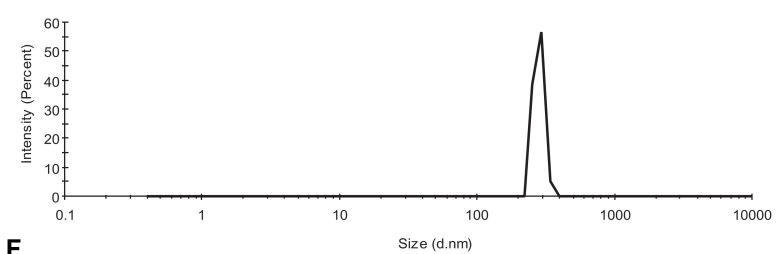

$\mathbf{F}$

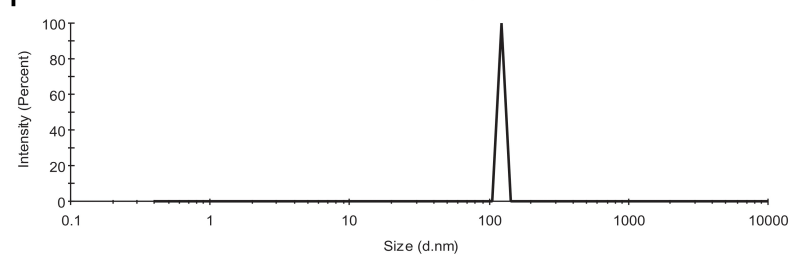

FIGURE 3 | Profile for dynamic light scattering (DLS) scattering of (A) untreated actin in PB; (B) actin treated with Ofloxacin in PB; (C) untreated actin control in GB; (D) actin treated with Ofloxacin in GB; (E) untreated actin control in water; and (F) treated actin with Ofloxacin at $300 \mu \mathrm{M}$.
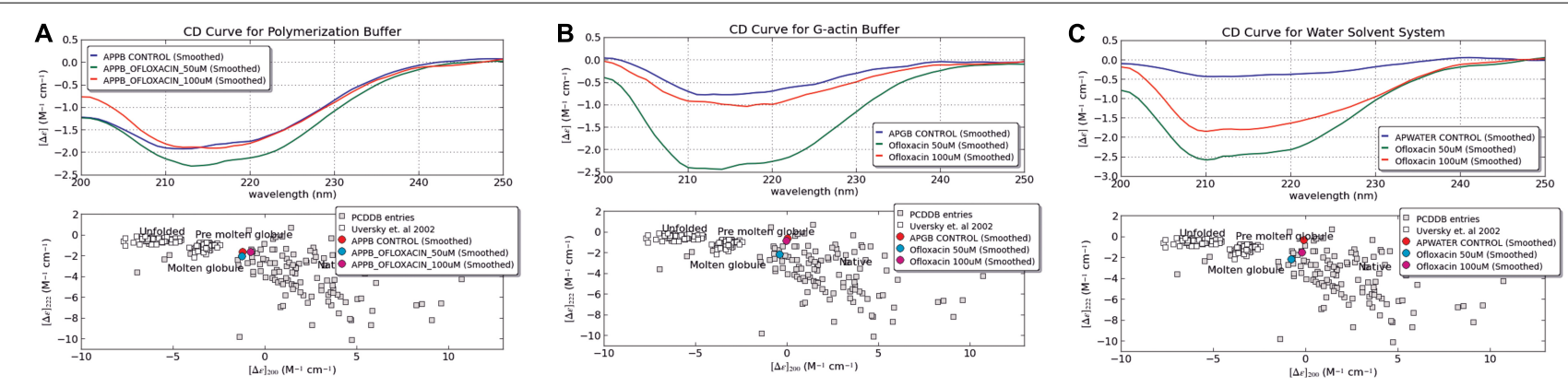

FIGURE 4 | CD Spectroscopic data analyzed for actin control and actin treated with Ofloxacin using CAPITO software. (A) PB; (B) GB; and (C) water. The blue curve is representative of actin control, whereas the green curve is representative of actin treated with Ofloxacin at $50 \mu \mathrm{M}$, and the red curve is representative of actin treated with Ofloxacin at $100 \mu \mathrm{M}$ in the respective buffer.

untreated and treated actin. Our BESTSEL analysis as observed in Figure 5A, is that actin control in polymerization buffer has $9.9 \%$ of $\alpha$-helix, $26.2 \beta$-sheet, $16.3 \%$ turns, and $45.5 \%$ other nonorganized structures. However, upon treatment with Ofloxacin in $\mathrm{PB}$ as observed in Figure 5B, the content of $\alpha$-helix was increased to $17.4 \%$, $\beta$-sheet was increased to $35.3 \%$, while turns and other unorganized structures were reduced to 13.3 and $33.4 \%$, respectively. Similarly, for the G-actin buffer, it was observed in Figures 5C,D that actin control had the presence of $\alpha$-helix of around 3.6\%, $\beta$-sheet, around $37.2 \%$, turn, $14.2 \%$, and other structures, $45.1 \%$. However, the treated actin had an $\alpha$-helix of around $18.3 \%, \beta$-sheet $37.6 \%$, turn, $9.5 \%$, and other structures, $34.7 \%$. Also, for actin control in water as observed in Figure 5E, we observed $\alpha$-helix to be around 4.5\%, $\beta$-sheet, $37.5 \%$, turn, $14.9 \%$, and other structures, $43.2 \%$. However, upon treatment, the structural change was reported to comprise $16 \%$ $\alpha$-helix, $25.3 \% \beta$-sheet, $12.4 \%$ turn, and $46.3 \%$ other unorganized structure as observed in Figure 5F. Our result of the BESTSEL analysis indicates that posttreatment of polymerized/aggregated actin resulted in actin enriched in $\alpha$-helical content which is likely to be closer to the globular actin molecule.

\section{SEM Imaging for the Actin Compound Interaction}

Scanning electron microscopy was performed for both treated and untreated actin in GB as well as water. PB was not used for these imaging studies due to the presence of high salt concentration in the buffer $(800 \mathrm{mM} \mathrm{KCl})$, which gave rise to more salt crystals to be observed in the SEM image. As observed in Figures 6a,c, untreated actin dialyzed against GB and water showed the presence of filamentous actin as well as 
A

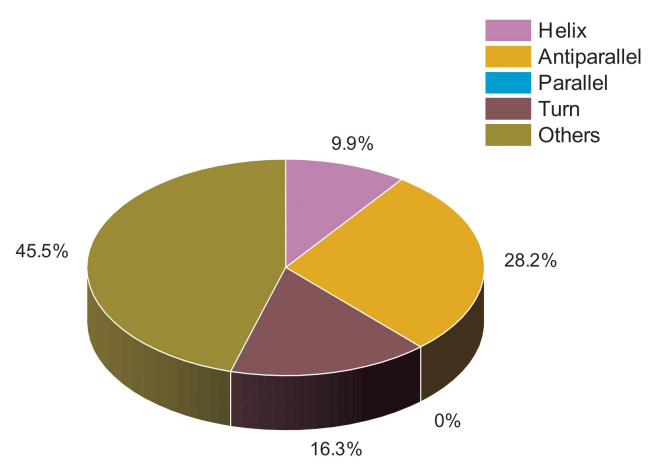

C

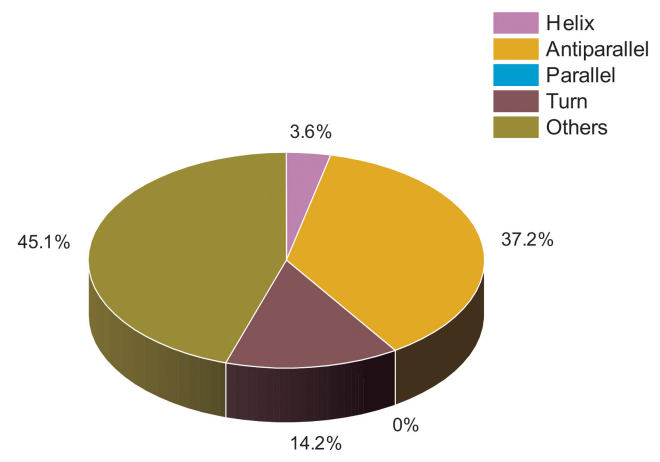

E

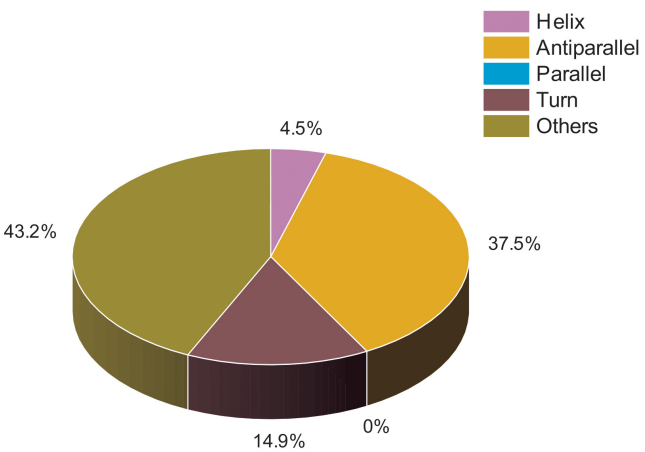

B

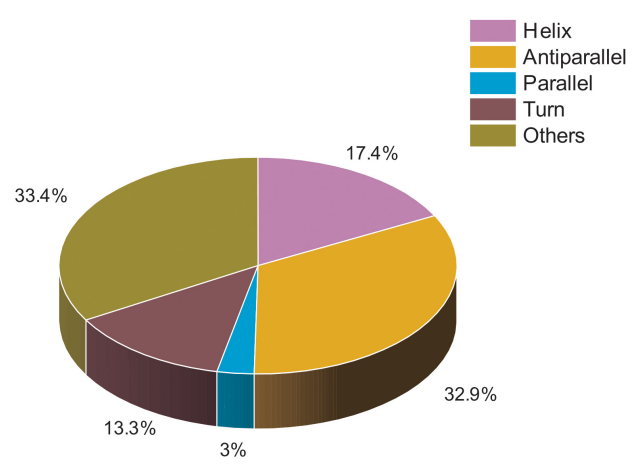

D

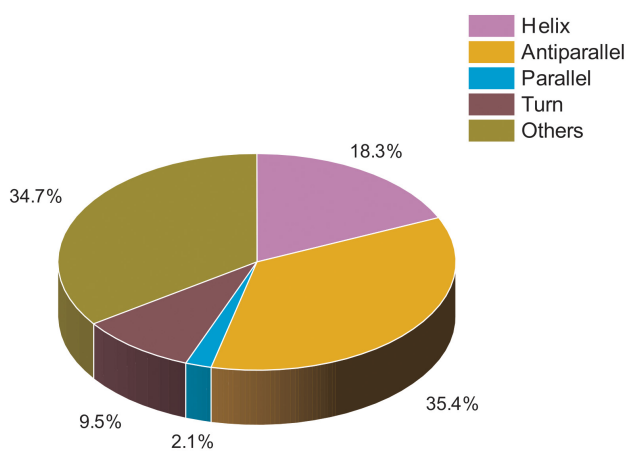

$\mathbf{F}$

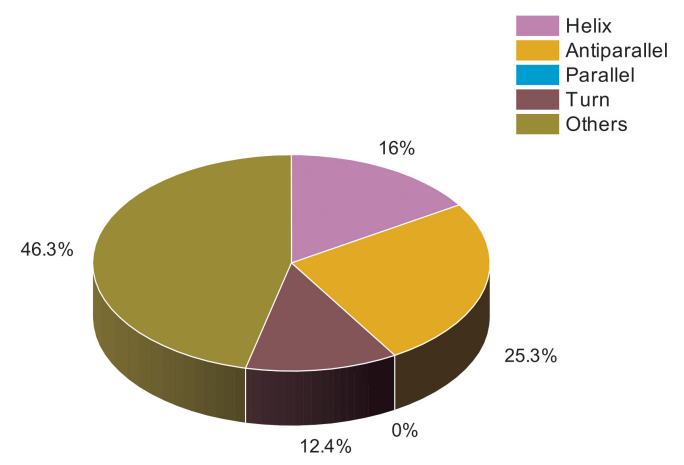

FIGURE 5 | Pie chart representation of the structural distribution of treated and untreated actin. (A) Actin control in PB. (B) Actin treated with Ofloxacin (50 $\mu$ M) in PB. (C) Actin control in GB. (D) Actin treated with Ofloxacin $(50 \mu \mathrm{M})$ in GB. (E) Actin control in water. (F) actin treated with Ofloxacin $(50 \mu \mathrm{M})$ in water.

amorphous actin aggregates, which when treated with Ofloxacin gets broken down to a smaller oligomeric protein as observed in Figures 6b,d.

\section{Kinetic Analysis of Actin Depolymerization}

We monitored the kinetic parameters for actin interactions with Ofloxacin in polymerization buffer as a function of right-angle scattering, which was measured every $5 \mathrm{~s}$ for up to $100 \mathrm{~min}$.
It is a known fact that right-angle scattering is proportionate to the size of the molecule. As observed in Figures 7A-I, the graph shows actin control in $\mathrm{PB}$ and the effect of Ofloxacin with its varying concentrations, which was analyzed and fit using a single exponential decay function of the kinetics. Three different parameters were reported through this fitting viz: (1) kagg, (2) amplitude (A2), and (3) y0. kagg stands for rate constant representing the speed of the reaction upon the interaction of actin polymer and Ofloxacin. A2 stands for amplitude indicating the amount of smaller actin oligomers formed upon 


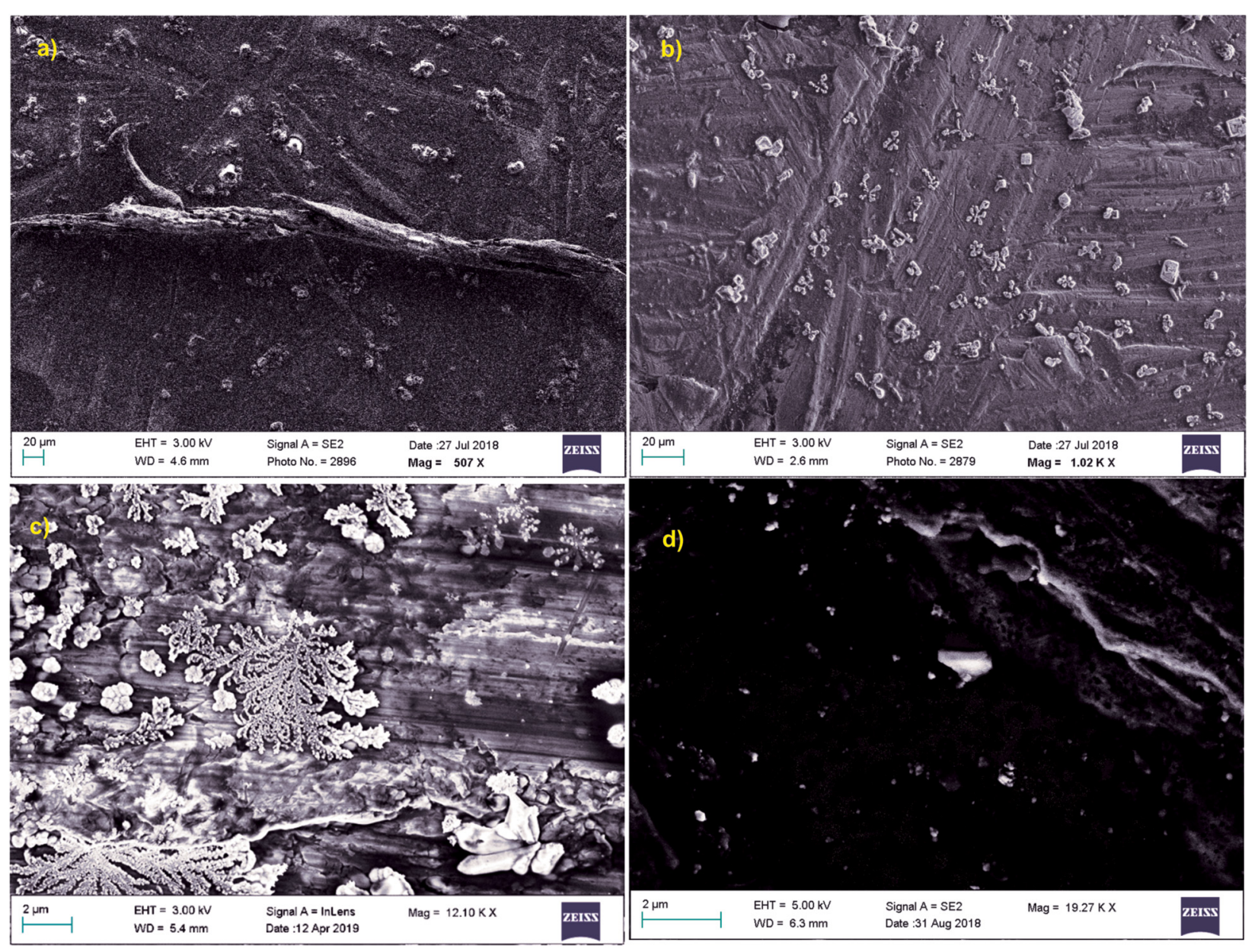

FIGURE 6 | SEM images for actin (a) dialyzed in G-actin buffer $20 \mu \mathrm{m}$; (b) treated with Ofloxacin in G-actin buffer $20 \mu \mathrm{m}$; (c) dialyzed in water $2 \mu \mathrm{M}$; and (d) treated with Ofloxacin in water $2 \mu \mathrm{M}$.

interaction with Ofloxacin. y0 stands for the extent to which the depolymerization has occurred upon interaction with the drug at the infinite $(\infty)$ time. The $R^{2}$ value for the single exponential decay fit was calculated to be between 0.97 and 0.72 as analyzed for varying concentrations of Ofloxacin interaction with actin.

The kinetics of polymerized F-actin in PB was measured for $100 \mathrm{~min}$ of its intrinsic polymerization and depolymerization dynamics that occur at the barbed end and the pointed end, respectively. We observed (Figures 7A, 8A) that actin control in polymerization buffer is quite stable and does not disintegrate substantially with the values for amplitude observed as 64.64 a.u., y0, 416.35 a.u., and t1, 1,820.99 s. However, upon treatment with Ofloxacin with a stoichiometric ratio of $1: 1$ as observed in Figure $\mathbf{7 B}$, the amplitude was calculated as 100.12 a.u., y0 was calculated as 271.80 a.u., and t1 was calculated as 2,693.98 s. With increasing concentration, a tremendous decrease in the intensity of the amplitude was observed indicating that Ofloxacin works to break down the highly aggregated actin into smaller oligomers. This process is concentration dependent. It was observed in our analysis that increasing concentration of Ofloxacin led to the decrease in the time required for breaking down the highly aggregated actin to smaller oligomers as well as the increase in the number of smaller oligomers formed. We also report the mode of interaction of the actin polymer with that of Ofloxacin, which follows a two-phased reaction. The first phase is quite faster than the second phase wherein an intermediate product is formed before the formation of the final product. The amplitude of the first phase (A1) can be calculated using Eq. (3) and is concentration dependent, while the second phase is not dependent upon the concentration of the Ofloxacin treatment.

Furthermore, we plotted the amplitude for the second phase reaction (A2), the time constant ( 11$)$, and $\mathrm{y} 0$ against the concentration of Ofloxacin. A graph of the extent of disintegration ( $\mathrm{y} 0$ ) against the concentration of Ofloxacin shows (Figure 8B) that as the concentration of the drug molecule increases, there is also an increase in the disintegrated product. We observed (Figure 8C) that the amount of the smaller oligomer formed is highest at a very high concentration of around $90 \mu \mathrm{M}$. Our graphical analysis for rate constant shows that the speed of interaction of Ofloxacin with that of actin polymer and 

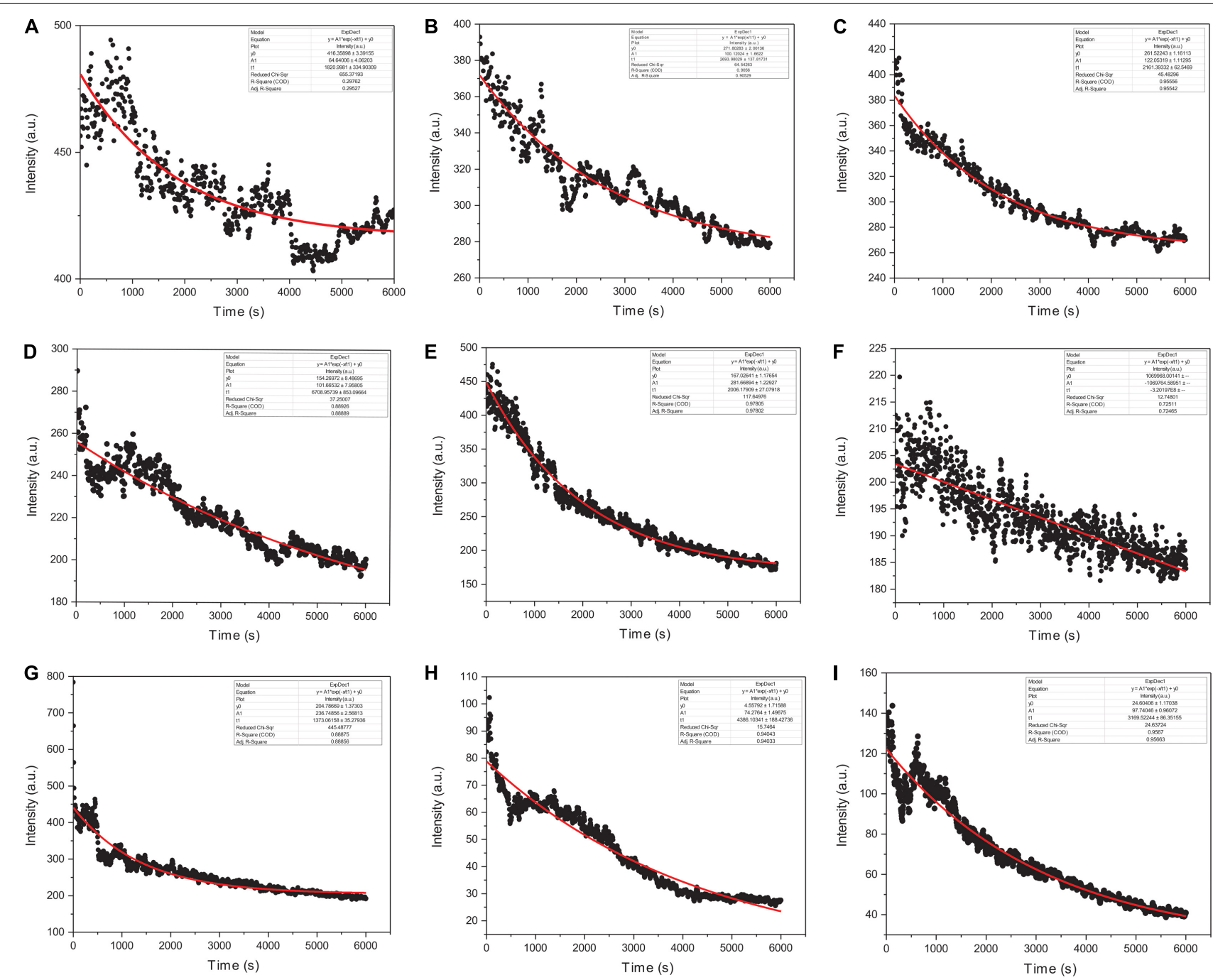

FIGURE 7 | Graphical representation of actin polymerization dynamics in the presence and absence of Ofloxacin at different concentrations observed in PB. (A) Actin control; (B) treated actin at $3 \mu \mathrm{M}$; (C) treated actin at $6 \mu \mathrm{M}$; (D) treated actin at $9 \mu \mathrm{M}$; (E) treated actin at $15 \mu \mathrm{M}$; (F) treated actin at $30 \mu \mathrm{M}$; (G) treated actin at $45 \mu \mathrm{M} ; \mathbf{( H )}$ treated actin at $60 \mu \mathrm{M}$; (I) treated actin at $90 \mu \mathrm{M}$. The red straight line indicates the single exponential curve fit analysis while the black dots represent the RLS intensity recorded every $5 \mathrm{~s}$ of actin-Ofloxacin interaction.

its subsequent breakdown increases with the increase in the concentration of Ofloxacin as observed in Figure 8D.

\section{Isothermal Titration Calorimetry Analysis for Actin Compound Interaction}

We carried out our interaction study of actin polymer and Ofloxacin using ITC in order to deduce the thermodynamic parameters for the reaction as well as the mode of binding. Figures 9A-D show the ITC profile for the actin aggregates interacting with Ofloxacin in polymerization buffer analyzed and tailored using a different model system. The upper panel represents the endothermic heat pulse with the first injection of around $0.4 \mu \mathrm{l}$ followed by 18 injections of $2 \mu \mathrm{l}$ each of $1.34 \mathrm{mM}$ Ofloxacin into the actin aggregate solution of $0.067 \mathrm{mM}$. The lower panel illustrates the integrated heat data indicative of the differential binding curve that was fit with using one-site binding, two-site sequential binding, threesite sequential binding, and four-site sequential binding model system. Although all the fit for the ITC data as seen in Table 1 shows binding to the actin of Ofloxacin strongly, the best fit was observed for the two-site sequential binding with the chi-square value of 1.373E5. The values of $\Delta H, \Delta S$, and $\mathrm{K}$ have been reported for all the four-binding models in Table 1. All of our binding fit shows that the reaction is both enthalpically as well as entropically driven. Two-site sequential binding of the drug molecule to the actin filament is enthalpically driven at both the sites of binding. This specifies that actin polymer/aggregate disruption upon binding of Ofloxacin is quite spontaneous and exothermic. Our data is indicative that Ofloxacin might be binding the actin polymer at multiple sites; however, two major sites on the actin polymer would 

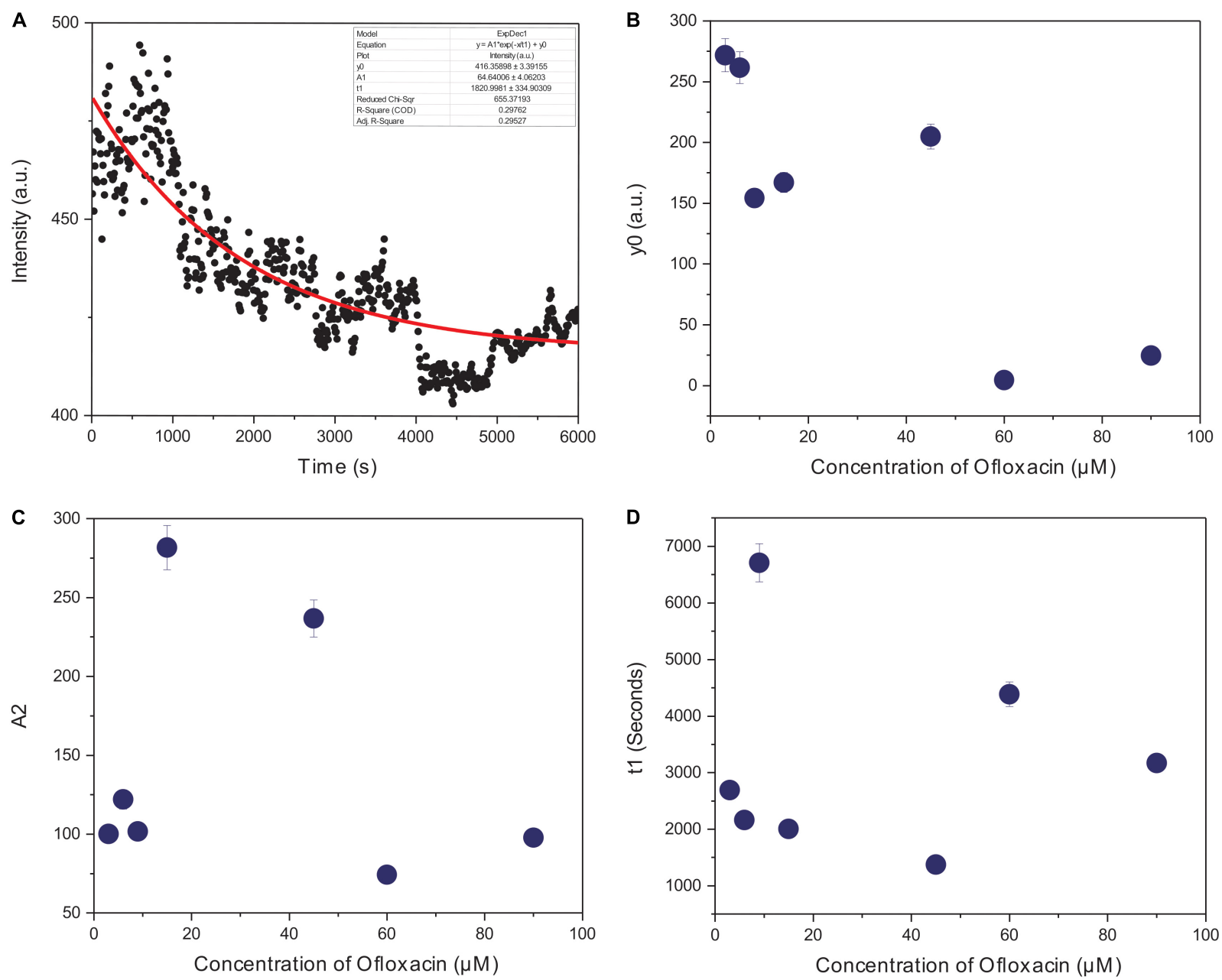

FIGURE 8 | Graphical representation of representative kinetic profiles in PB for actin treated with Ofloxacin: (A) Actin control; (B) extent of disintegration (y0); (C) amplitude (A2); and (D) time constant (t1).

be more favorable for Ofloxacin binding than the rest of the other binding sites.

\section{In silico Data Analysis}

We performed autodocking studies for hexameric actin interaction with Ofloxacin in order to deduce the possible mode of binding of the drug molecule to that of the filament. We observed in Figure $\mathbf{1 0}$ that most of the clusters of the Ofloxacin drug bind to the lateral interface of the actin timer. Various previous studies have suggested that actin during nucleation forms a rigid nucleus known for producing a critical concentration to promote the process of elongation of the filament (Cooper and Schafer, 2000; Vavylonis et al., 2005; Bugyi and Carlier, 2010; Blanchoin et al., 2014; Cell Signaling, 2014; De La Cruz and Gardel, 2015; Galkin et al., 2015; Coutts and La Thangue, 2016; Carlier and Shekhar, 2017). Our docking studies suggest that Ofloxacin tries to either inhibit by binding to the lateral interface as observed for Cluster 1, Site 2, Site 3, and Site 4 or disrupts the already formed nuclei in the system.
It has also been observed in our data that the drug molecule is also getting associated with SD-2 of actin, which is rich in coiled coil as is prevalent in Cluster 2. It is an already known fact that these SD-2 of the actin monomer are responsible for the major conformational changes that drive the dynamics of actin polymerization (Blanchoin et al., 2014; Galkin et al., 2015; Carlier and Shekhar, 2017). We speculate that actin might be undergoing major conformational change upon Ofloxacin binding at SD-2, thereby, disintegrating the actin molecule. This data is quite in consensus with our ITC where the thermodynamics of the interaction reaction supports two-site sequential binding of the drug to actin.

\section{DISCUSSION}

Ofloxacin is one of the widely used broad-spectrum fluoroquinolone antibiotic used against several bacterial infections such as bronchitis, pneumonia, chlamydia, gonorrhea, skin infections, urinary tract infections, and infections of 

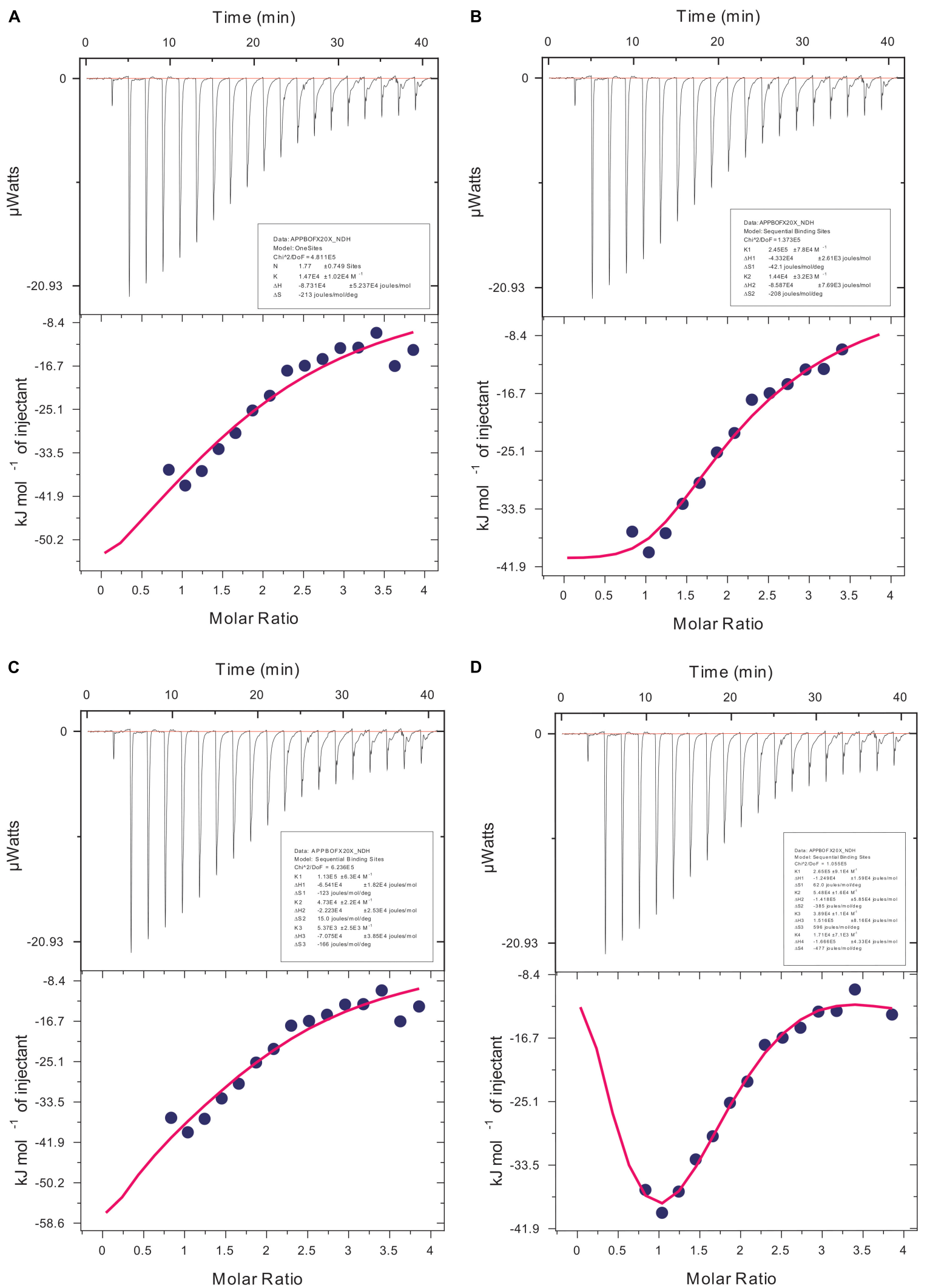

FIGURE 9 | Isothermal calorimetric profile for actin aggregates treated with Ofloxacin in PB. (A) Model: one-site binding; (B) model: sequential two-site binding; (C) model: sequential three-site binding; and (D) model: sequential four-site binding. 
TABLE 1 | Thermodynamic parameters obtained using isothermal titration calorimetry (ITC) for Ofloxacin.

\begin{tabular}{|c|c|c|c|c|}
\hline Parameters & One-site binding & Sequential two-site binding & Sequential three-site binding & Sequential four-site binding \\
\hline $\mathrm{Chi}^{\wedge} 2 / \mathrm{DoF}$ & 4.811 E5 & 1.373E5 & 6.236E5 & 1.055E5 \\
\hline K1 & $1.47 \mathrm{E} 4 \pm 1.02 \mathrm{E} 4 \mathrm{M}^{-1}$ & $2.45 \mathrm{E} 5 \pm 7.8 \mathrm{E} 4 \mathrm{M}^{-1}$ & $1.13 \mathrm{E} 5 \pm 6.3 \mathrm{E} 4 \mathrm{M}^{-1}$ & $2.65 \mathrm{E} 5 \pm 9.1 \mathrm{E} 4 \mathrm{M}^{-1}$ \\
\hline$\Delta \mathrm{H} 1$ & $-8.731 \mathrm{E} 4 \pm 5.237 \mathrm{E} 4 \mathrm{~J} / \mathrm{mol}$ & $-4.332 \mathrm{E} 4 \pm 2.61 \mathrm{E} 3 \mathrm{~J} / \mathrm{mol}$ & $-6.541 \mathrm{E} 4 \pm 1.82 \mathrm{E} 4 \mathrm{~J} / \mathrm{mol}$ & $-1.249 \mathrm{E} 4 \pm 1.59 \mathrm{E} 4 \mathrm{~J} / \mathrm{mol}$ \\
\hline$\Delta \mathrm{S} 1$ & $-213 \mathrm{~J} / \mathrm{mol} / \mathrm{deg}$ & $-42.1 \mathrm{~J} / \mathrm{mol} / \mathrm{deg}$ & $-123 \mathrm{~J} / \mathrm{mol} / \mathrm{deg}$ & $62.0 \mathrm{~J} / \mathrm{mol} / \mathrm{deg}$ \\
\hline K2 & & $1.44 \mathrm{E} 4 \pm 3.2 \mathrm{E} 3 \mathrm{M}^{-1}$ & $4.73 \mathrm{E} 4 \pm 2.2 \mathrm{E} 4 \mathrm{M}^{-1}$ & $5.48 \mathrm{E} 4 \pm 1.6 \mathrm{E} 4 \mathrm{M}^{-1}$ \\
\hline$\Delta \mathrm{H} 2$ & & $-8.587 \mathrm{E} 4 \pm 7.69 \mathrm{E} 3 \mathrm{~J} / \mathrm{mol}$ & $-2.223 \mathrm{E} 4 \pm 2.53 \mathrm{E} 4 \mathrm{~J} / \mathrm{mol}$ & $-1.418 \mathrm{E} 5 \pm 5.85 \mathrm{E} 4 \mathrm{~J} / \mathrm{mol}$ \\
\hline$\Delta \mathrm{S} 2$ & & $-208 \mathrm{~J} / \mathrm{mol} / \mathrm{deg}$ & $15.0 \mathrm{~J} / \mathrm{mol} / \mathrm{deg}$ & $-385 \mathrm{~J} / \mathrm{mol} / \mathrm{deg}$ \\
\hline K3 & & & $5.37 \mathrm{E} 3 \pm 2.5 \mathrm{E} 3 \mathrm{M}^{-1}$ & $3.89 \mathrm{E} 4 \pm 1.1 \mathrm{E} 4 \mathrm{M}^{-1}$ \\
\hline$\Delta \mathrm{H3}$ & & & $-7.075 \mathrm{E} 4 \pm 3.85 \mathrm{E} 4 \mathrm{~J} / \mathrm{mol}$ & $1.516 \mathrm{E} 5 \pm 8.16 \mathrm{E} 4 \mathrm{~J} / \mathrm{mol}$ \\
\hline$\Delta \mathrm{S3}$ & & & $-166 \mathrm{~J} / \mathrm{mol} / \mathrm{deg}$ & $596 \mathrm{~J} / \mathrm{mol} / \mathrm{deg}$ \\
\hline K4 & & & & $1.71 \mathrm{E} 4 \pm 7.1 \mathrm{E} 3 \mathrm{M}^{-1}$ \\
\hline$\Delta \mathrm{H} 4$ & & & & $-1.666 \mathrm{E} 5 \pm 4.33 \mathrm{E} 4 \mathrm{~J} / \mathrm{mol}$ \\
\hline$\Delta S 4$ & & & & $-477 \mathrm{~J} / \mathrm{mol} / \mathrm{deg}$ \\
\hline
\end{tabular}

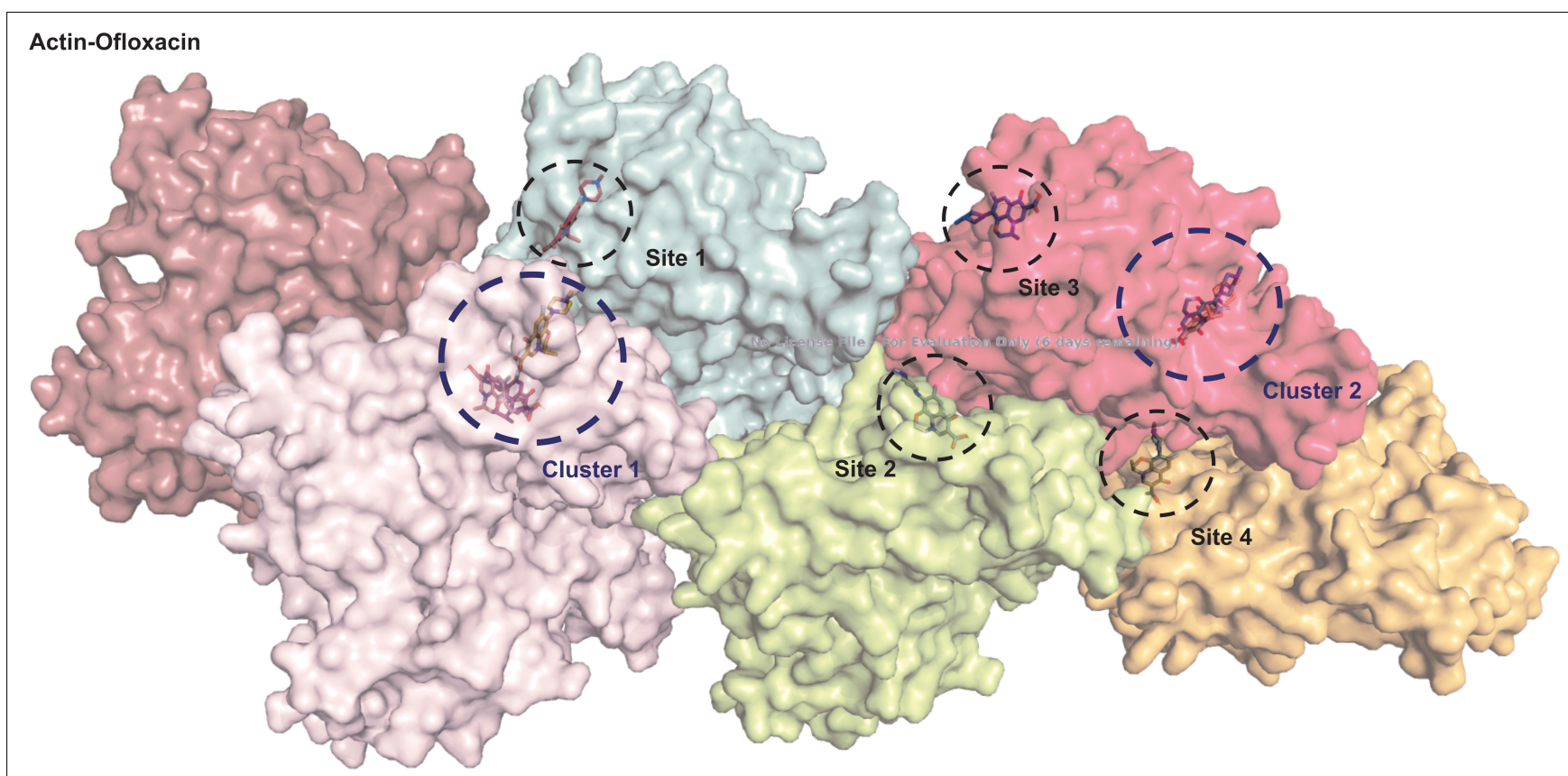

FIGURE 10 | Actin polymer interaction with Ofloxacin. Hexamer actin. ChainA is shown salmon, ChainB in purple, ChainC in cyan, ChainD in lemon, ChainE in pink, and ChainF in orange.

the prostate (Administration, n.d.; Kern et al., 1987; Eron and Gentry, 1992). Drug repositioning is a new approach that has been emerging recently for the treatment of various diseases as any lead molecule under a de novo drug discovery program, which takes around 10-15 years to come into the market and probably has a success rate of less than $10 \%$. It has been a known fact that the FDA has approved different molecules against 400 human proteins (Pillaiyar et al., 2020). These proteins are classified under the umbrella of enzymes, transporters, G protein-coupled receptors (GPCRs), cluster of differentiation (CD) markers, voltage-gated ion channels, and nuclear receptors (Pillaiyar et al., 2020). Actin is one of the globular proteins with an ATPase binding cleft and an intrinsic property to polymerize (Oda et al., 2009; McKayed and Simpson, 2013). This polymerization dynamics that has been regulated by various actin-binding protein forms the driving force for many cellular processes such as cellular motility, cellular niche formation, and transport of biological molecules (Shekhar et al., 2016; Carlier and Shekhar, 2017). It has been observed that aberrations in the actin polymerization-depolymerization cycle lead to aggregation of the actin molecule leading to various diseased conditions (Lambrechts et al., 2004; Fletcher and Mullins, 2010; Muñoz-Lasso et al., 2020b). The unregulated actin dynamics has been implicated into the various neuropathological conditions, which manifest with the aging process among 
humans (Lambrechts et al., 2004; Gourlay and Ayscough, 2005; Goebel, 2009; Yao and Khan, 2012).

These neuropathological conditions are difficult to treat and lead to a decline in the quality of life. Thus, there is a widespread need for molecules with low cell toxicity to be identified for its ability to control and monitor cytoskeletal protein especially actin whose pathology has been implicated in arising cases of neurological apathies. Our current study emphasizes upon identifying a molecule, which can depolymerize the actin aggregates to smaller oligomers and prevent subsequent neuronal cell death that arises due to these aggregate formations. We utilized various biophysical techniques to study the role of Ofloxacin, a broad-spectrum antibiotic with a very low level of cell toxicity on actin polymerization-depolymerization dynamics. We also utilized in silico docking study in order to understand the mechanism of binding of Ofloxacin to actin and its subsequent role in breaking down the highly amorphous or polymerized actin to smaller aggregates.

Owing to the fact that human actin is closer to pig (S. scrofa) actin, we purified actin from the acetone powder of pig thigh muscle. The protein was purified upon several polymerization and depolymerization cycles in $\mathrm{PB}$ buffer rich in salt concentration and GB buffer, which has the presence of a reducing agent and ATP. The purified protein was isolated and separated at $42-\mathrm{kDa}$ molecular weight on the SDS page. It was also confirmed using the excised band for mass spectrometric analysis to be actin. This protein was then used for further interaction studies with the drug Ofloxacin. We performed constant wavelength synchronous analysis to observe for the scattering profile for the three morphological states of actin in $\mathrm{PB}, \mathrm{GB}$, and water and the drug molecule in $\mathrm{PB}$. This data was measured for up to $48 \mathrm{~h}$. The measured data for the actin in three different buffers had the presence of high scattering intensity from 250 to $700 \mathrm{~nm}$ indicating toward the fact that all three morphological states of the actin remain in the filamentous, oligomeric, and aggregated state. However, when we did the same analysis for the drug, it was observed that actin shows negligible scattering up to $395 \mathrm{~nm}$ beyond, which starts self-aggregating resulting in a very high scattering intensity from 400 to $700 \mathrm{~nm}$. Hence, for our further rightangle scattering measurement, we used $350 \mathrm{~nm}$. Furthermore, we carried out a right-angle scattering measurement for actinOfloxacin interaction with varying concentrations of the drug molecule up to $48 \mathrm{~h}$. We observed through our RLS measurement that actin controls in all the three buffer systems, which was having very high scattering intensity due to the presence of a higher molecular weight aggregate that gets disrupted to a smaller oligomeric size with low scattering intensity as the function of the concentration of Ofloxacin. This binding and disruption in all the three buffer systems were irreversible as no increase in the scattering intensity was observed posttreatment even after the $48 \mathrm{~h}$ of incubation.

We performed dynamic light measurement in order to deduce the particle size obtained posttreatment with Ofloxacin. It was observed that the heterogenous peak representing variations in shape and size of the actin aggregate in three different solvent systems was transformed to a homogenous peak of a smaller size indicating the breakdown of the actin polymer/aggregate to a smaller oligomeric or monomeric state. The structural change in the actin morphology pre- and posttreatment was analyzed using the $\mathrm{CD}$ spectrometric measurement, the data for which was collected in mdeg. The data for the untreated actin control as well as the treated actin in the three buffer systems were analyzed both manually using two different software viz: CAPITO (Wiedemann et al., 2013) and BESTSEL (Micsonai et al., 2018). The information obtained through our analysis indicated an increase in the structural population of the $\alpha$-helix in treated actin compared to their untreated counterparts. The oligomers/monomers obtained posttreatment had a structural component closer to the content of globular actin, which is also rich in $\alpha$-helix (Pathak et al., 2020). Actin in PB is more polymeric or filamentous with highly exposed hydrophobic residues and has near-native compactness, while actin in GB is more globular with native compactness of the protein and substantially a higher $\alpha$-helix structure. This distinct feature of GB is due to the fact that this buffer is capable of disrupting the actin aggregate/polymer into a smaller oligomeric size of the actin. At a lower concentration of Ofloxacin, around $25 \mu \mathrm{M}$, we do not observe much change in the structural content from that of the treated actin. Thus, we choose to have the concentration of the drug molecule with a ratio of 1:10 and above. One of the important findings of our CD data using our CAPITO analysis was that treated actin in PB and GB brings about a concentrationdependent change from molten globule to that of the more globular structure. On the contrary, actin in water, which occurs as an amorphous aggregate, was seen to be occurring as a globular structure. Treated actin at $50 \mu \mathrm{M}$ occurs as a molten globular structure with a more exposed hydrophobic structure with near-native compactness (Perera et al., 2016). As soon as the concentration is increased to $100 \mu \mathrm{M}$. actin changes from a molten globular structure to a more globular structure. This change, which is typical of water, is because of the amorphous aggregate that has a more unorganized structure, which is more spherical and compact, with relatively less exposed hydrophobic structure and a detectable tertiary structure. However, at a lower concentration of Ofloxacin, actin is disrupted as a more linear polymeric or large oligomeric secondary structure occurring as a molten globule with near-native compactness. At the increased concentration of $100 \mu \mathrm{M}$, actin is disrupted to monomeric or lower oligomeric structure rich in $\alpha$-helix with near-native compactness and less exposed hydrophobic residues. Although we tried to increase the concentration of Ofloxacin with a ratio up to 1:50, it leads to the saturation of the $\mathrm{CD}$ detector.

We then also perform imaging studies to observe for the morphological difference in the treated and untreated actin. Control actin dialyzed against GB and water shows the presence of filamentous actin as well as amorphous actin aggregate. However, upon treatment with Ofloxacin, this highly filamentous as well as amorphous aggregate is converted to the morphology of smaller oligomers and monomers. We also performed the kinetic study for the actin-Ofloxacin interaction as a function of rightangle scattering measured against the varying concentrations of Ofloxacin. We tried to deduce three basic parameters from our study viz, $\mathrm{y} 0$, which represents the extent to which disintegration 
occurs, the time constant (t1), which is the time taken to break down and stabilize the reaction, as well as amplitude (A2), which represents the number of lower oligomers formed posttreatment with actin in polymerization buffer. Our analyzed data revealed that actin itself undergoes constant recycling of polymerization and depolymerization as a result of its intrinsic property as was observed for the actin control in PB. However, upon the treatment, we deduced that the amount of actin oligomeric content (A2) increased with an increase in the concentration of Ofloxacin, thereby, decreasing the content of highly aggregated actin in the system with each increasing concentration. We also see that actin polymer/aggregate upon treatment with Ofloxacin follows a two-phased reaction of which the first phase of the reaction remains tremendously fast compared to the second phase, which is relatively very slow. We observed that an intermediate product is formed before the formation of the end product in our kinetic analysis. The time required to stabilize the reaction was as short as $30 \mathrm{~min}$, although our measurement was carried out up to $100 \mathrm{~min}$ and more. Most of the interaction was found to occur in the first phase, which is concentration dependent beyond which, as we enter the second phase post the intermediate product formation, actin disruption is independent of the concentration of Ofloxacin.

We then studied our interaction of actin-Ofloxacin in $\mathrm{PB}$ using ITC to deduce the thermodynamic parameters for the reaction and its mode of binding. Although our result shows binding for one site and multiple site sequential binding, the best fit was observed for two-site binding with low error values indicating that there are two major sites on actin polymer/aggregate, which favors the binding of Ofloxacin and subsequent disintegration of the actin molecule to a lower oligomeric size. We also observed that, on both of these sites, the interaction is enthalpically as well as entropically driven. Our in silico data shows two prevalent sites for binding of Ofloxacin to that of actin hexamer. These sites include the lateral interface, which is important for actin monomer interaction to form the nuclei, and the other site is near SD-2. We speculate that actin might undergo conformation change in its three-dimensional lattice upon Ofloxacin binding at SD-2 as well as inhibits the interaction of the actin monomer at the interface, which are responsible for nuclei formation as shown in Figure 11. This data agrees with our ITC data, which show a preferential mode of binding to the two-site sequential binding. The plausible mechanism that we have deduced is that Ofloxacin binds the actin at the aforementioned sites viz, cluster 1 and cluster 2, thereby, bringing about conformational change and destabilizing the larger aggregates. This is followed by the disintegration of large actin aggregates into the oligomeric structure. The accumulation of smaller nuclei or monomeric actin might prevent the obliteration of neuronal cells due to the inclusion bodies of actin.

\section{CONCLUSION}

In the current study, Ofloxacin, which is a widely used broadspectrum antibiotic for various bacterial infections, is elucidated as a potential candidate for drug repurposing. We studied actin aggregate as a significant therapeutic target site to treat various neurodegenerative as well as neurodevelopmental disorders. In order to study the role that Ofloxacin plays on these protein molecules, we purified actin from the pig thigh muscle (S. scrofa domesticus) in three different solvent systems. The three solvent systems, namely, $\mathrm{PB}, \mathrm{GB}$, and water mimic the in vivo morphology of the actin protein inside a human cell. These result in the actin being purified as a long filamentous polymer when dialyzed against $\mathrm{PB}$, as smaller oligomers when dialyzed against $\mathrm{GB}$, whereas in water, it formed amorphous aggregates. Pig thigh muscle was used as it is highly homologous with that of human actin with a $95 \%$ identity. Throughout the initial high-throughput screening of Ofloxacin on different morphologies of actin, we observed that the drug molecule was indeed disrupting large actin molecules.

In order to understand the actual role of Ofloxacin on actin aggregates, we performed CWSF analysis. During this assay,

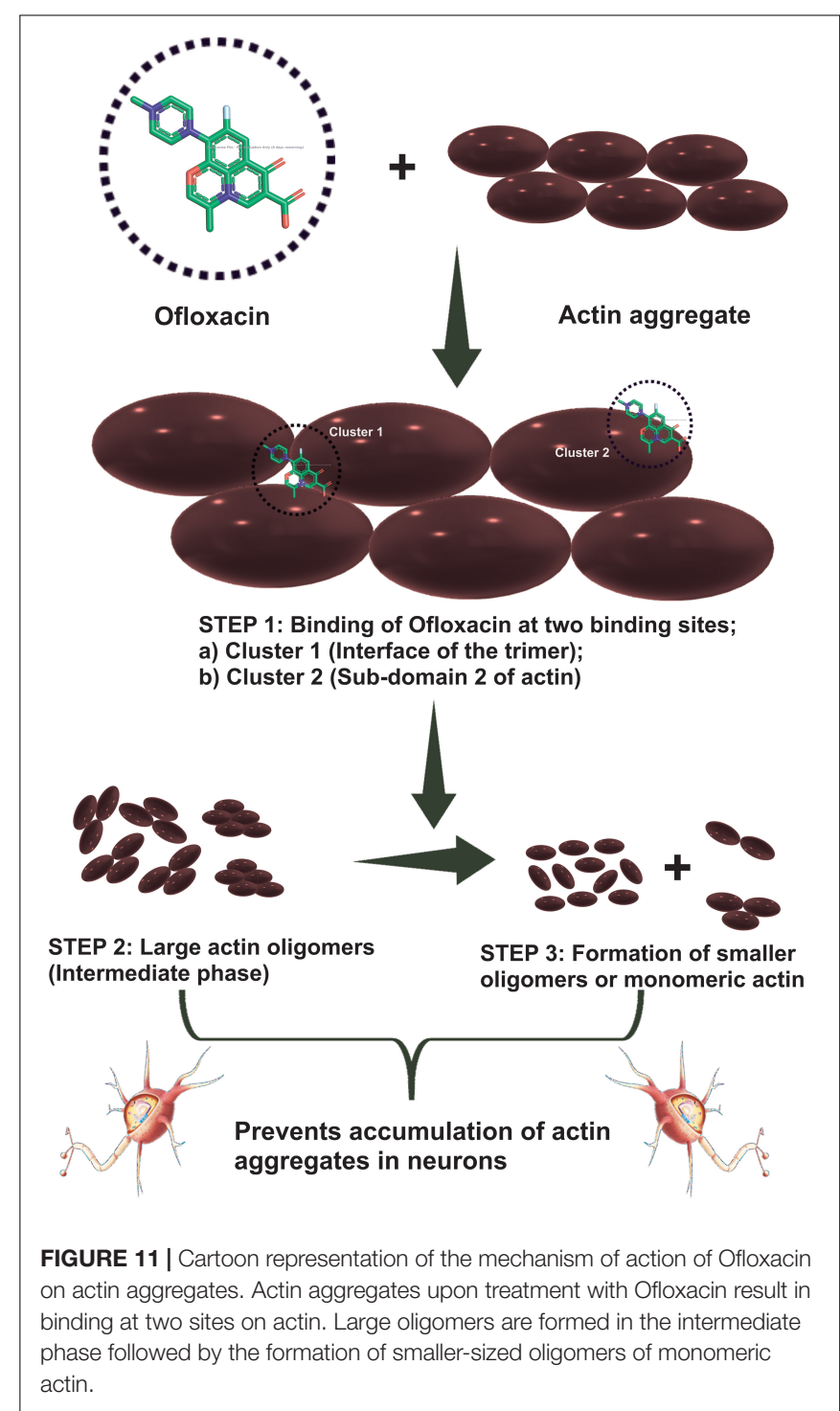


we observed that actin in all the three buffer systems shows higher scattering from 250 to $700 \mathrm{~nm}$ for $48-72 \mathrm{~h}$ without any drops. This indicates that actin remains as a large molecular-sized polymer, oligomer, or amorphous aggregate for a long period of time. We then performed the same assay for the drug up to $72 \mathrm{~h}$, which resulted in Ofloxacin showing scattering post $400 \mathrm{~nm}$ wavelength. In order to avoid any major interference from the drug molecule during our RLS measurement, we used a wavelength of $350 \mathrm{~nm}$ as the scattering of the drug up to $390 \mathrm{~nm}$ remains negligible. During the right-angle scattering, we observed that there was a concentration-dependent drop in the scattering intensity of the actin molecule in all the three solvent systems. Since this drop in the intensity was observed up until $72 \mathrm{~h}$, we concluded that Ofloxacin causes an irreversible disruption of larger actin aggregates. We then performed DLS, which resulted in the convergence of heterogenous peaks of actin aggregates in $\mathrm{PB}, \mathrm{GB}$, and water to a homologous peak of smaller sizes indicating the change in the actin morphology and abundance of either smaller actin oligomers or monomers.

We also performed CD spectroscopic analysis in all the threebuffer systems, which indicates a shift of a highly polymeric structure of untreated actin into an actin rich in $\alpha$-helix posttreatment. This analysis leads us to conclude that differential actin aggregate changed to globular actin. In order to observe the morphological changes in actin, we also performed SEM analysis. Through our data, we reported that the disintegration of actin occurred in both GB and water upon treatment with Ofloxacin. Our kinetic data analysis of Ofloxacin interaction with that of actin polymer in polymerization buffer indicates that the disruption of larger actin aggregates followed a two-phase reaction. During the first phase of the reaction, which is much faster than the second phase, a large molecule of actin aggregate is formed into a smaller actin nuclei, which is further disrupted into a smaller oligomer or monomeric actin. The second phase of the reaction, which is relatively slow, does not show much change in activity upon the increase in the concentration of Ofloxacin. We thus concluded that only the first phase of the reaction, which forms the intermediate product of actin aggregates, is concentration dependent. Isothermal calorimetry data suggested that the best binding occurred with two set

\section{REFERENCES}

Abraham, M. J., Murtola, T., Schulz, R., Páll, S., Smith, J. C., Hess, B., et al. (2015). Gromacs: high-performance molecular simulations through multi-level parallelism from laptops to supercomputers. SoftwareX 1-2, 19-25. doi: 10. 1016/j.softx.2015.06.001

Blanchoin, L., Boujemaa-Paterski, R., Sykes, C., and Plastino, J. (2014). Actin dynamics, architecture, and mechanics in cell motility. Physiol. Rev. 94, 235-263. doi: 10.1152/physrev.00018. 2013

Borana, M. S., Mishra, P., Pissurlenkar, R. R. S., Hosur, R. V., and Ahmad, B. (2014). Curcumin and kaempferol prevent lysozyme fibril formation by modulating aggregation kinetic parameters. Biochim. Biophys. Acta 1844, 670-680. doi: 10.1016/j.bbapap.2014.01.009

Bourne, J. N., and Harris, K. M. (2008). Balancing structure and function at hippocampal dendritic Spines. Annu. Rev. Neurosci. 31, 47-67. doi: 10.1146/annurev.neuro.31.060407. sequential bindings with the interaction being enthalpically and entropically driven. The interaction was further studied using in silico analysis of actin-drug association. It was understood from the auto-dock data that Ofloxacin binds to actin at two major sites viz, Sub-domain 2 as well as at the interface of the actin nuclei. Ofloxacin was supposedly found to interfere with both the longitudinal as well as lateral interactions between two associating actin monomers.

Through our biophysical and mechanistic understanding of actin-Ofloxacin interaction, we suggest that the drug could be used as a potential candidate against the prognosis and treatment of several neurodegenerative as well as a neurodevelopmental disorders. In order to make it to the market, much further work in the area of drug dosage and drug delivery of this molecule is required. A molecular vehicle, either organic or nanomaterial, capable of encapsulating the drug as well as crossing the BBB would prove to be beneficial in targeting the dysregulated actin aggregate formed in the neuronal cells.

\section{DATA AVAILABILITY STATEMENT}

All datasets presented in this study are included in the article/supplementary material.

\section{AUTHOR CONTRIBUTIONS}

SP executed the experiment, analyzed the data, and wrote the manuscript. HP executed the experiment and wrote the manuscript. ST executed the experiments. AK conceptualized the work, analyzed the data, and wrote the manuscript. All authors contributed to the article and approved the submitted version.

\section{FUNDING}

We would like to acknowledge Department of Biotechnology (DBT), Government of India (Grant number: BT/PR16325/NER/ 95/117/2015) for partial financial support.

Bugyi, B., and Carlier, M. -F. (2010). Control of actin filament treadmilling in cell motility. Annu. Rev. Biophys. 39, 449-470. doi: 10.1146/annurev-biophys051309-103849

Carlier, M. F., and Shekhar, S. (2017). Global treadmilling coordinates actin turnover and controls the size of actin networks. Na. Rev. Mol. Cell Biol. 18, 389-401. doi: 10.1038/nrm.2016.172

Cell Signaling (2014). Regulation of Actin Dynamics. Danvers, MA: Cell Signaling Technology, Inc.

Chanfreau, G. F., Gourlay, C. W., Carpp, L. N., Timpson, P., Winder, S. J., Ayscough, K. R., et al. (2005). Impact and prevention of neurodegenerative diseases in society: alzheimer and Parkinson. Trends Cell Biol. 118:460.

Chavan, R., Mukherjee, S., Dahake, R., Colvin, D., Kale, A., and Chowdhary, A. (2016). Differential proteomic analysis of respiratory samples from patients suffering from influenza. VirusDisease 27, 226-233. doi: 10.1007/s13337-0160332-x

Cooper, J. A., and Schafer, D. A. (2000). Control of actin assembly and disassembly at filament ends. Curr. Opin. Cell Biol. 12, 97-103. doi: 10.1016/S0955-0674(99) 00062-9 
Coutts, A. S., and La Thangue, N. B. (2016). Regulation of actin nucleation and autophagosome formation. Cell. Mol. Life Sci. 73, 3249-3263. doi: 10.1007/ s00018-016-2224-z

De La Cruz, E. M., and Gardel, M. L. (2015). Actin mechanics and fragmentation. J. Biol. Chem. 290, 17137-17144. doi: 10.1074/jbc.R115.636472

Debreczeni, J. É, and Emsley, P. (2012). ). Handling ligands with Coot. Acta Crystallogr. Sect. D 68, 425-430. doi: 10.1107/S0907444912000200

Eira, J., Silva, C. S., Sousa, M. M., and Liz, M. A. (2016). The cytoskeleton as a novel therapeutic target for old neurodegenerative disorders. Prog. Neurobiol. 141, 61-82. doi: 10.1016/j.pneurobio.2016.04.007

Emsley, P., Lohkamp, B., Scott, W. G., and Cowtan, K. (2010). Features and development of Coot. Acta Crystallogr. Sect. D 66, 486-501. doi: 10.1107/ S0907444910007493

Eron, L. J., and Gentry, L. O. (1992). Oral ofloxacin for infections caused by bacteria resistant to oral antimicrobial agents. Diagn. Microbiol. Infect. Dis. 15, 435-439. doi: 10.1016/0732-8893(92)90085-8

Feigin, V. L., Nichols, E., Alam, T., Bannick, M. S., Beghi, E., Blake, N., et al. (2019). Global, regional, and national burden of neurological disorders, 1990-2016: a systematic analysis for the Global Burden of Disease Study 2016. Lancet Neurol. 18, 459-480. doi: 10.1016/S1474-4422(18)30499-X

Fletcher, D. A., and Mullins, R. D. (2010). Cell mechanisms and cytoskeleton. Nature 463, 485-492. doi: 10.1038/nature08908.Cell

Food and Drug Administration (n.d.). FLOXIN Tablets, Vol. 7. 1-32. Avaliable at: https://www.accessdata.fda.gov/drugsatfda_docs/label/2007/019735s057lbl. pdf (accessed September, 2008).

Fukazawa, Y., Saitoh, Y., Ozawa, F., Ohta, Y., Mizuno, K., and Inokuchi, K. (2003). Hippocampal LTP is accompanied by enhanced F-actin content within the dendritic spine that is essential for late LTP maintenance in vivo. Neuron 38 , 447-460. doi: 10.1016/s0896-6273(03)00206-x

Furgerson, M., Fechheimer, M., and Furukawa, R. (2012). Model hirano bodies protect against tau-independent and tau-dependent cell death initiated by the amyloid precursor protein intracellular domain. PLoS One 7:e0044996. doi: 10.1371/journal.pone.0044996

Furukawa, R., and Fechheimer, M. (1997). The structure, function, and assembly of actin filament bundles. Int. Re. Cytol. 175, 29-90. doi: 10.1016/s0074-7696(08) 62125-7

Galkin, V. E., Orlova, A., Vos, M. R., Schröder, G. F., and Egelman, E. H. (2015). Near-atomic resolution for one state of F-Actin. Structure 23, 173-182. doi: 10.1016/j.str.2014.11.006

Gammon, K. (2014). Neurodegenerative disease: brain windfall. Nature 515, 299 300. doi: 10.1038/nj7526-299a

Gitler, A. D., Dhillon, P., and Shorter, J. (2017). Neurodegenerative disease: models, mechanisms, and a new hope. DMM Dis. Models Mech. 10, 499-502. doi: $10.1242 / \mathrm{dmm} .030205$

Goebel, H. H. (2009). Protein aggregate myopathies: introduction. Brain Pathol. 19, 480-482. doi: 10.1111/j.1750-3639.2009.00291.x

Goldman, J. E. (1983). The association of actin with hirano bodies. J. Neuropathol. Exp. Neurol. 42, 146-152. doi: 10.1097/00005072-198303000-00004

Gordon-Weeks, P. R., and Fournier, A. E. (2014). Neuronal cytoskeleton in synaptic plasticity and regeneration. J. Neurochem. 129, 206-212. doi: 10.1111/ jnc. 12502

Gourlay, C. W., and Ayscough, K. R. (2005). The actin cytoskeleton in ageing and apoptosis. FEMS Yeast Res. 5, 1193-1198. doi: 10.1016/j.femsyr.2005.08.001

Kern, W., Kurrle, E., and Vanek, E. (1987). Ofloxacin for prevention of bacterial infections in granulocytopenic patients. Infection 15, 427-432. doi: 10.1007/ BF01647222

Kevenaar, J. T., and Hoogenraad, C. C. (2015). The axonal cytoskeleton: from organization to function. Front. Mol. Neurosci. 8:44. doi: 10.3389/fnmol.2015. 00044

Kim, C., and Lisman, J. E. (1999). A role of actin filament in synaptic transmission and long-term potentiation. J. Neurosci. 19, 4314-4324. doi: 10.1523/jneurosci. 19-11-04314.1999

Koistinen, N. A., Edlund, A. K., Menon, P. K., Ivanova, E. V., Bacanu, S., and Iverfeldt, K. (2017). Nuclear localization of amyloid- $\beta$ precursor proteinbinding protein Fe65 is dependent on regulated intramembrane proteolysis. PLoS One 12:e0173888. doi: 10.1371/journal.pone.0173888

Lambrechts, A., Van Troys, M., and Ampe, C. (2004). The actin cytoskeleton in normal and pathological cell motility. Int. J. Biochem. Cell Biol. 36, 1890-1909. doi: 10.1016/j.biocel.2004.01.024
Liu, H., Pierre-Pierre, N., and Huo, Q. (2012). Dynamic light scattering for gold nanorod size characterization and study of nanorod-protein interactions. Gold Bull. 45, 187-195. doi: 10.1007/s13404-012-0067-4

Luo, L. (2002). Actin cytoskeleton regulation in neuronal morphogenesis and structural plasticity. Annu. Rev. Cell Dev. Biol. 18, 601-635. doi: 10.1146/ annurev.cellbio.18.031802.150501

Maselli, A., Furukawa, R., Thomson, S. A. M., Davis, R. C., and Fechheimer, M. (2003). Formation of hirano bodies induced by expression of an actin crosslinking protein with a gain-of-function mutation. Eukaryotic Cell 2, 778-787. doi: $10.1128 /$ ec.2.4.778-787.2003

McKayed, K., and Simpson, J. (2013). Actin in action: imaging approaches to study cytoskeleton structure and function. Cells 2, 715-731. doi: 10.3390/ cells 2040715

Melidone, R., Keating, J. H., Pfannl, R., and Alroy, J. (2011). Cerebral hirano-like bodies in an alpaca (Vicugna pacos). Vet. Pathol. 49, 723-726. doi: 10.1177/ 0300985811406886

Micsonai, A., Wien, F., Bulyáki, É, Kun, J., Moussong, É, Lee, Y. H., et al. (2018). BeStSel: a web server for accurate protein secondary structure prediction and fold recognition from the circular dichroism spectra. Nucleic Acids Res. 46, W315-W322. doi: 10.1093/nar/gky497

Morrissey, I., Hoshino, K., Sato, K., Yoshida, A., Hayakawa, I., Bures, M. G., et al. (1996). Mechanism of differential activities of ofloxacin enantiomers? Antimicrob. Agents nd Chemother. 40, 1775-1784. doi: 10.1128/aac.40.8.1775

Muñoz-Lasso, D. C., Mollá, B., Calap-Quintana, P., García-Giménez, J. L., Pallardo, F. V. V., Palau, F., et al. (2020a). Cofilin dysregulation alters actin turnover in frataxin-deficient neurons. Sci. Rep. 10, 1-10. doi: 10.1038/s41598-020-62050-7

Muñoz-Lasso, D. C., Romá-Mateo, C., Pallardó, F. V., and Gonzalez-Cabo, P. (2020b). Much more than a scaffold: cytoskeletal proteins in neurological disorders. Cells 9:358. doi: 10.3390/cells9020358

Oda, T., Iwasa, M., Aihara, T., Maéda, Y., and Narita, A. (2009). The nature of the globular- to fibrous-actin transition. Nature 457, 441-445. doi: 10.1038/ nature07685

Ogawa, Y., and Rasband, M. N. (2008). The functional organization and assembly of the axon initial segment. Curr. Opin. Neurobiol. 18, 307-313. doi: 10.1016/j. conb.2008.08.008

Okada, H., and Soderling, S. H. (2009). Signaling through actin to regulate spine formation and function. Open Neurosci. J. 3, 97-107. doi: 10.2174/ 1874082000903020097

Otterbein, L. R., Graceffa, P., and Dominguez, R. (2001). The crystal structure of uncomplexed actin in the ADR state. Science 293, 708-711. doi: 10.1126/science. 1059700

Pathak, S., Tripathi, S., Deori, N., Ahmad, B., Verma, H., Lokhande, R., et al. (2020). Effect of tetracycline family of antibiotics on actin aggregation, resulting in the formation of Hirano bodies responsible for neuropathological disorders. J. Biomol. Struct. Dyn. 1-18. doi: 10.1080/07391102.2020.1717629 [Epub ahead of print].

Pelucchi, S., Stringhi, R., and Marcello, E. (2020). Dendritic spines in alzheimer's disease: how the actin cytoskeleton contributes to synaptic failure. Int. J. Mol. Sci. 21, 1-23. doi: 10.3390/ijms21030908

Perera, S. P., McIntosh, T. C., and Wanasundara, J. P. D. (2016). Structural properties of cruciferin and napin of brassica napus (Canola) show distinct responses to changes in ph and temperature. Plants 5:36. doi: 10.3390/ plants5030036

Perez-Iratxeta, C., and Andrade-Navarro, M. A. (2008). K2D2: estimation of protein secondary structure from circular dichroism spectra. BMC Struct. Biol. 8:25. doi: 10.1186/1472-6807-8-25

Perl, D. P., Davidson, M., Haroutunian, V., and Davis, K. L. (1995). Alzheimer's disease and related neurodegenerative diseases in elderly schizophrenic patients. J. Neuropathol. Exp. Neurol. 54:460. doi: 10.1097/00005072199505000-00211

Pillaiyar, T., Meenakshisundaram, S., and Manickam, M. (2020). A medicinal chemistry perspective of drug repositioning: recent advances and challenges in drug discovery. Eur. J. Med. Chem. 195:112275. doi: 10.1016/j.ejmech.2020. 112275

Przedborski, S., Vila, M., and Jackson-Lewis, V. (2003). Neurodegeneration: what is it and where are we? J. Clin. Invest. 111, 3-10. doi: 10.1172/JCI200317522

Pushpakom, S., Iorio, F., Eyers, P. A., Escott, K. J., Hopper, S., Wells, A., et al. (2019). Drug repurposing: progress, challenges and recommendations. Nat. Rev. Drug Discov. 18, 41-58. doi: 10.1038/nrd.2018.168 
Ross, C. A., and Poirier, M. A. (2004). Protein aggregation and neurodegenerative disease. Nat. Med. 10:S10. doi: 10.1038/nm1066

Sabo, S. L., Ikin, A. F., Buxbaum, J. D., and Greengard, P. (2001). The Alzheimer amyloid precursor protein (APP) and FE65, an APP-binding protein, regulate cell movement. J. Cell Biol. 153, 1403-1414. doi: 10.1083/jcb.153.7. 1403

Santa-Mara, I., Santpere, G., MacDonald, M. J., Gomez De Barreda, E., Hernandez, F., Moreno, F. J., et al. (2008). Coenzyme Q induces tau aggregation, tau filaments, and Hirano bodies. J. Neuropathol. Exp. Neurol. 67, 428-434. doi: 10.1097/NEN.0b013e31816fc9b6

Shekhar, S., Pernier, J., and Carlier, M.-F. (2016). Regulators of actin filament barbed ends at a glance. J. Cell Sci. 129, 1085-1091. doi: 10.1242/jcs.179994

Solomon, E. I., Augustine, A. J., and Yoon, J. (2010). Rapid and efficient purification of actin from nonmuscle sources. Cell Motil. Cytoskeleton 39, 3921-3932. doi: $10.1039 / \mathrm{b} 800799 \mathrm{c} . \mathrm{O}$

Sonavane, S., Haider, S. Z., Kumar, A., and Ahmad, B. (2017). Hemin is able to disaggregate lysozyme amyloid fibrils into monomers. Biochim. Biophys. Acta 1865, 1315-1325. doi: 10.1016/j.bbapap.2017.07.017

Spears, W., Furgerson, M., Sweetnam, J. M., Evans, P., Gearing, M., Fechheimer, M., et al. (2014). Hirano bodies differentially modulate cell death induced by tau and the amyloid precursor protein intracellular domain. BMC Neurosci. 15:74. doi: 10.1186/1471-2202-15-74

Spence, E. F., and Soderling, S. H. (2015). Actin out: regulation of the synaptic cytoskeleton. J. Biol. Chem. 290, 28613-28622. doi: 10.1074/jbc.R115. 655118

Subramaniam, S. (2019). Selective neuronal death in neurodegenerative diseases: the ongoing mystery. Yale J. Biol. Med. 92, 695-705.

Svennberg, K. (2006). Moisture Buffering in the Indoor Environment. Physics 67, 184-192. doi: 10.1016/j.brainresrev.2011.01.003.Impaired

Szabó, E. C., Manguinhas, R., and Fonseca, R. (2016). The interplay between neuronal activity and actin dynamics mimic the setting of an LTD synaptic tag. Sci. Rep. 6, 1-14. doi: 10.1038/srep33685
Trends, C. M., and Disorders, N. (2018). Current medication trendsand global impact on neurodegenerative disorders. J. Pharmaceut. Pharmacol. 6, 01-09. doi: $10.13188 / 2327-204 x \cdot 1000024$

Trott, O., and Olson, A. J. (2010). AutoDock Vina: improving the speed and accuracy of docking with a new scoring function, efficient optimization, and multithreading. J. Comput. Chem. 31, 455-461. doi: 10.1002/jcc.21334

Vavylonis, D., Yang, Q., and O'Shaughnessy, B. (2005). Actin polymerization kinetics, cap structure, and fluctuations. Proc. Natl. Acad. Sci. U.S.A. 102, 8543-8548. doi: 10.1073/pnas.0501435102

Wiedemann, C., Bellstedt, P., and Görlach, M. (2013). CAPITO - A web serverbased analysis and plotting tool for circular dichroism data. Bioinformatics 29, 1750-1757. doi: 10.1093/bioinformatics/btt278

Yamada, R., and Kuba, H. (2016). Structural and functional plasticity at the axon initial segment. Front. Cell. Neurosci. 10:250. doi: 10.3389/fncel.2016.00250

Yang, G., Sau, C., Lai, W., Cichon, J., and Li, W. (2010). ADF/cofilin-actin rods in neurodegenerative diseases J.R. Curr. Alzheimer Res. 7, 241-250. doi: 10.1126/ science.1249098.Sleep

Yao, J., and Khan, A. N. (2012). Involvement of actin pathology in Alzheimer's Disease. Cell Dev. Biol. 02, 2-4. doi: 10.4172/2168-9296.1000e121

Yao, J., and Khan, A. N. (2013). Involvement of actin pathology in Alzheimer's Disease. Cell Dev. Biol. 02, 2-4.

Conflict of Interest: The authors declare that the research was conducted in the absence of any commercial or financial relationships that could be construed as a potential conflict of interest.

Copyright $\odot 2020$ Pathak, Parkar, Tripathi and Kale. This is an open-access article distributed under the terms of the Creative Commons Attribution License (CC BY). The use, distribution or reproduction in other forums is permitted, provided the original author(s) and the copyright owner(s) are credited and that the original publication in this journal is cited, in accordance with accepted academic practice. No use, distribution or reproduction is permitted which does not comply with these terms. 